\title{
1 Functional specialization of monocot DCL3 and DCL5 proteins through the
}

\section{2 evolution of the PAZ domain}

3

4 Shirui Chen ${ }^{1,2}$, Wei Liu ${ }^{1,2}$, Masahiro Naganuma ${ }^{1,3}$, Yukihide Tomari ${ }^{1,2, *}$, Hiro-oki 5 Iwakawa $^{1, *}$

6

$7 \quad{ }^{1}$ Institute for Quantitative Biosciences, The University of Tokyo, Bunkyo-ku, Tokyo 113-

80032 , Japan

$9{ }^{2}$ Department of Computational Biology and Medical Sciences, Graduate School of

10 Frontier Sciences, The University of Tokyo, Bunkyo-ku, Tokyo 113-0032, Japan

113 Present address, RIKEN Center for Biosystems Dynamics Research, Yokohama,

12 Kanagawa, 230-0045, Japan

$13{ }^{*}$ Correspondence: tomari@iqb.u-tokyo.ac.jp (Y.T.), iwakawa@iqb.u-tokyo.ac.jp (H.-o.I.)

14 


\section{$15 \underline{\text { Abstract }}$}

16 Monocot DICER-LIKE3 (DCL3) and DCL5 produce distinct 24-nt heterochromatic

17 small interfering RNAs (hc-siRNAs) and phased secondary siRNAs (phasiRNAs). The

18 former small RNAs are linked to plant heterochromatin, and the latter to reproductive

19 processes. It is assumed that these DCLs evolved from an ancient "eudicot-type" DCL3

20 ancestor, which may have produced both types of siRNAs. However, how functional

21 differentiation was achieved after gene duplication remains elusive. Here, we find that

22 monocot DCL3 and DCL5 exhibit biochemically distinct preferences for 3' overhangs

23 and $5^{\prime}$ phosphates, consistent with the structural properties of their in vivo double-

24 stranded RNA substrates. Importantly, these distinct substrate specificities are determined

25 by the PAZ domains of DCL3 and DCL5 which have accumulated mutations during the

26 course of evolution. These data explain the mechanism by which these DCLs cleave their

27 cognate substrates from a fixed end, ensuring the production of functional siRNAs. Our

28 study also indicates how plants have diversified and optimized RNA silencing

29 mechanisms during evolution. (157 words) 


\section{$31 \quad$ Introduction}

32 Small interfering RNAs (siRNAs) and microRNAs (miRNAs) are critical players in RNA

33 silencing pathways which regulate various biological processes including organismal

34 development and antiviral immunity ${ }^{1-4}$. These small RNAs are processed from either long

35 double-stranded RNAs (dsRNAs) or RNAs with hairpin-like structures by specific

36 ribonucleases called Dicer in animals or Dicer-like (DCL) proteins in plants ${ }^{5,6}$. These

37 Dicer and DCL proteins are evolutionary conserved multidomain proteins belonging to

38 the RNase III family ${ }^{6}$. While mammals have a single Dicer, plants encode multiple DCL

39 proteins that produce different types of small RNAs ${ }^{7}$. For example, the genome of the

40 model plant Arabidopsis thaliana encodes four DCL proteins, AtDCL1-4 with precise

41 activities. AtDCL1 produces 20 to 22-nucleotide (nt) miRNAs, while AtDCL4 and 2

42 generate 21 and 22-nt siRNAs, respectively ${ }^{7}$. These small RNAs then regulate protein

43 and mRNA levels through post-transcriptional gene silencing ${ }^{7}$. In contrast, AtDCL3

44 produces heterochromatic $24-n t$ siRNAs (hc-siRNAs) that form specific RNA-induced silencing complexes (RISCs) with ARGONAUTE4/6 (AGO4/6). RISCs promote

46 sequence-specific DNA methylation and thus transcriptional gene silencing ${ }^{8}$. This RNA-

47 directed DNA Methylation (RdDM) process is essential in repressing transposable

48 elements, responding to stresses and maintaining genome integrity ${ }^{9-11}$. In short, the

49 evolution of DCL proteins has led to diverse mechanisms that regulate gene expression

50 at different levels.

AtDCL3 targets dsRNAs that are generated by the sequential action of two 
53 polymerase 2 (RDR2) $)^{12-15}$. Pol IV synthesizes 30-40-nt RNAs (Pol IV strand), which

54 often bear adenine at the $5^{\prime}$ end ${ }^{16,17}$. RDR2 then synthesizes the complementary strand of

55 the Pol IV strand (RDR2 strand) through its RNA-dependent RNA polymerase activity ${ }^{16}$.

56 The resulting dsRNAs are called Pol IV and RDR2-dependent RNAs (P4R2 RNAs) ${ }^{18}$.

57 RDR2 tends to add one or two non-templated nucleotide(s) to the 3 ' end of the RDR2

58 strand via its terminal nucleotidyl transferase activity. Thus, P4R2 RNAs typically harbor

59 a 1- or 2-nt 3' overhang on the RDR2 strand, while the 3' end of Pol IV-generated strands

60 are blunt-ended (Figure 1A, left panel) ${ }^{16,18}$. Interestingly, AtDCL3 preferentially cleaves

61 P4R2 RNAs from the $5^{\prime}$ end of the Pol IV strand ${ }^{17}$. This asymmetric dicing by AtDCL3

62 is thought to be achieved by the combination of $5^{\prime}$ A selection upon Pol IV transcription

63 and preference for the unstable $5^{\prime} \mathrm{A}$ and $\mathrm{U}$ end resulting from AtDCL3 cleavage ${ }^{17,19}$.

64 However, it remains unclear if the difference in thermodynamic stability alone explains

65 the biased cleavage.

Although nascent transcripts of RNA polymerases generally have a

67 triphosphate group at the $5^{\prime}$ end ${ }^{15}$, the P4R2 RNAs that accumulate in $d c l$ mutant plants

68 and mature hc-siRNAs both have a $5^{\prime}$ monophosphate group ${ }^{17,18,20}$. One explanation for

69 this is that DCL3 cleaves P4R2 RNAs after the tri- to monophosphate conversion of the

$70 \quad 5^{\prime}$ end through an unknown activity ${ }^{17,21}$. In contrast, a recent biochemical analysis showed

71 that recombinant AtDCL3 cleaves not only substrates harboring a 5' monophosphate, but

72 also those with a $5^{\prime}$ triphosphate ${ }^{16}$. This suggests that AtDCL3 can directly cleave P4R2

73 RNAs with $5^{\prime}$ triphosphates in vivo. 
siRNAs are called reproductive phased secondary 24-nt siRNAs (24-nt phasiRNA),

76 which are highly expressed in anthers ${ }^{23,24}$. Generally, phasiRNAs are produced from the RNAs targeted by 22-nt small RNAs ${ }^{25-28}$. The 22-nt small RNA-loaded Argonaute (AGO) proteins cleave the target RNA, resulting in the production of a 3' cleavage fragment with a $5^{\prime}$ monophosphate. This fragment is then converted into a dsRNA with a triphosphate at the $5^{\prime}$ end of the antisense strand by RDR6, which is recruited via SILENCING DEFECTIVE 5 (SDE5) and the complex consisting of 22-nt small RNA, ARGONAUTE1 (AGO1) and SUPPRESSOR OF GENE SILENCING 3 (SGS3) ${ }^{29}$. Because RDR6 begins RNA synthesis at the third nucleotide of the template's $3^{\prime}$ end ${ }^{30}$, the Pol II (sense) strand of the dsRNA has 2-nt 3' overhang (Figure 1A, right panel). In contrast, the $3^{\prime}$ end of the RDR6 (antisense) strand of the dsRNA is more heterogeneous, having a blunt end or bearing a 1-nt or 2-nt non-templated nucleotide added by the terminal nucleotidyl transferase activity of RDR6 (Figure 1A, right panel) ${ }^{30-32}$. The dsRNA intermediate is then processed by DCLs into phasiRNAs, with the phase determined by the small RNAguided cleavage site. The mechanism of this one-way processing remains unclear. In monocots, DCL5, which is thought to have evolved via duplication of DCL3 (also called DCL3b), specifically produces 24-nt phasiRNAs in the anther (Supplementary Figure 1) ${ }^{33}$. Although eudicots are believed to lack the 24-nt phasiRNA pathway, recent studies argue that some eudicots like Citrus sinensis and Populus trichocarpa produce 24-nt

94 phasiRNAs even without encoding DCL5 (Supplementary Figure 1) ${ }^{22,28}$. In these plants,

95 DCL3 needs to produce both 24-nt phasiRNAs and hc-siRNAs. Given the completely 
97 and monocot and eudicot DCL3 need to tailor their substrate specificities to their cognate

98 targets. However, so far, the biochemical properties of these DCLs, which are relevant to

99 gene regulation and reproduction, have not been examined and compared.

100 Dicer and DCL proteins generally consist of five functional domains: the

101 helicase domain, PAZ (PIWI, AGO, and Zwille) domain, two RNase III domains and

102 double-stranded RNA-binding domain from $\mathrm{N}$ to $\mathrm{C}$ terminal ${ }^{6,34,35}$. Previous biochemical

103 and structural studies of human DICER1 and Drosophila melanogaster Dicer-2 (Dcr-2)

104 demonstrated that the PAZ domain has two pockets that bind the $5^{\prime}$ and $3^{\prime}$ ends of the

105 substrate dsRNA respectively. These binding pockets are critical for the precise

106 production of small $\mathrm{RNAs}^{36-38}$. In addition to the PAZ domain, it is reported that the

107 helicase domain interacts with the substrate dsRNA and is required for Drosophila Dcr-2

108 to bind the $3^{\prime}$ end ${ }^{39-41}$. However, because the sequences of PAZ and helicase domains of

109 plant DCLs greatly differ from human Dicer and Drosophila Dcr-2 ${ }^{42}$, the mechanisms

110 underlying substrate recognition and specific catalysis by plant DCLs remain unclear.

111 In this study, we succeeded in preparing fully functional recombinant eudicot

112 AtDCL3, monocot Oryza sativa DCL3 (OsDCL3) and DCL5 (OsDCL5). Our analysis

113 elucidates how DCL3 and DCL5 have become functionally specialized after gene

114 duplication. OsDCL5 and OsDCL3 have distinct substrate specificities for both 5'

115 phosphate and $3^{\prime}$ structures, reflecting the different in vivo functions of these proteins.

116 Moreover, we find that the PAZ domain is a key determinant of DCL3 and DCL5

117 substrate specificity. These preferences explain how DCL3 and DCL5 cleave substrates

118 from the fixed end to ensure the production of functional siRNAs. Importantly, eudicot 
119 AtDCL3 has intermediate preferences between OsDCL5 and OsDCL3 for both a blunt

120 ended structure and $5^{\prime}$ triphosphate. This argues that OsDCL5 and OsDCL3 became

121 functionally specialized after duplication from the "eudicot-type" DCL3. Taken together,

122 our study provides insights into the functional differentiation of DCLs via the evolution

123 of the PAZ domains. This provides a molecular understanding of how plants have

124 diversified and optimized RNA silencing mechanisms through DCL gene duplication. 


\section{$126 \underline{\text { Results }}$}

127 DCL5 and DCL3 proteins have different preferences for $\mathbf{3}^{\prime}$ dsRNA structures.

128 To compare the substrate preferences of monocot DCL5 and monocot and eudicot DCL3

129 proteins in vitro, we successfully prepared full-length recombinant DCL proteins:

130 OsDCL5, OsDCL3, and AtDCL3 using Drosophila S2 cells (Figure 1B). Double-

131 stranded RNA (dsRNA) substrates were radiolabeled at the 5' end of the sense or antisense

132 strand and incubated with purified recombinant DCL for the in vitro dicing assay. Each

133 strand of the substrate was $38 \mathrm{nt}$ long, thus mimicking the length of P4R2 RNAs. Since

134 dsRNAs can be cleaved from both ends, two product bands (24-nt and 16-nt) were

135 expected (Figure 1C). The cleavage efficiency was calculated by dividing the sum of the

136 cleaved fragments by the total amount of the substrate (full-length + cleaved fragments).

137 To determine the 3' structures preferred by DCL3 and DCL5 proteins respectively, we

138 performed in vitro dicing assays with dsRNAs harboring different 3 ' structures: blunt end

139 (BLT), 1-nt overhang (1ovr), and 2-nt overhang (2ovr) (Figure 2A). Both AtDCL3 and

140 OsDCL3 cleaved dsRNAs with overhangs more efficiently than the BLT substrates

141 (Figure 2B, C). At early time points (5-30 minutes after incubation), OsDCL3 showed a

142 more pronounced preference for overhangs than AtDCL3 (Figure 2B, C). In contrast,

143 OsDCL5 cleaved BLT, 1ovr and 2ovr dsRNA substrates with similar efficiency (Figure

144 2D), showing no specific preference for $3^{\prime}$ structures. Interestingly, both AtDCL3 and

145 OsDCL3 generated multiple cleavage products from BLT substrates (Figure 2B, C). We

146 speculate that AtDCL3 and OsDCL3 cannot accurately process BLT substrates, resulting

147 in intermediate products that are longer than 24-nt. These intermediate products might 
148 then be cleaved again, generating fragments shorter than 16-nt (Figure 2B, C). In contrast,

149 OsDCL5 cleaved BLT as accurately as 1ovr and 2ovr substrates. Taken together, we

150 conclude that DCL5, monocot and eudicot DCL3 proteins have different preferences for

151 the $3^{\prime}$ structure of dsRNAs; OsDCL3 has the strongest preference for $3^{\prime}$ overhangs,

152 followed by AtDCL3, while OsDCL5 has no apparent preference for specific 3' structures.

154 The $5^{\prime}$ phosphate of dsRNAs is required for efficient cleavage by both DCL3 and 155 DCL5.

156 In addition to the recognition of the $3^{\prime}$ structure, the recognition of the $5^{\prime}$ end of RNA 157 substrates is also important for both accurate and efficient dicing of dsRNAs ${ }^{39,43}$. Previous 158 in vitro dicing assays using crude plant lysates confirmed that a $5^{\prime}$ phosphate is required 159 for AtDCL3-mediated cleavage of dsRNAs carrying $3^{\prime}$ overhangs ${ }^{19}$. To investigate the 160 importance of the 5' phosphate of dsRNAs in DCL3- and DCL5-mediated cleavage, we 161 performed in vitro dicing assays with $3^{\prime} 1$-nt overhang substrates radiolabeled at the $5^{\prime}$ 162 monophosphate of antisense strands. These substrates carry either a $5^{\prime}$ monophosphate 163 group (MonoP) or a hydroxyl group $(\mathrm{OH})$ on the sense strand (Figure 3A). If the 5' 164 monophosphate is required for substrate processing, a 5'-hydroxyl should decrease the 165 generation of $15-n t$ cleavage products which arise from the $5^{\prime}$ end of the sense strand. We 166 found that, for all three DCL proteins, 15-nt products generated from a 5'-OH substrate 167 were decreased compared to MonoP substrates (Figure 3B-D. This result argues that the $1685^{\prime}$ phosphate of the substrate is required for efficient dsRNA cleavage by both DCL3 and DCL5. 


\section{DCL3 and DCL5 have distinct preferences for a 5' triphosphate on the dsRNA.}

172 In theory, newly synthesized RNAs generated by Pol IV and RDR2 carry 5 ' triphosphates.

173 It is therefore possible that P4R2 RNAs carry a 5' triphosphate when they encounter

174 DCL3. In addition, precursors of phasiRNAs, i.e. DCL5 substrates, are also likely to

175 possess a triphosphate group at the $5^{\prime}$ end of the antisense strand, which is synthesized by

176 RDR6. To investigate the effect of a $5^{\prime}$ triphosphate group DCL3 and DCL5-mediated

177 cleavage, we performed in vitro dicing assays with dsRNA substrates carrying a 5'-32 $\mathrm{P}$ on

178 the antisense strand. Substrates were monophosphorylated (MonoP) or triphosphorylated

179 (TriP) at the $5^{\prime}$ end of their sense strands. Since cleavage from the $5^{\prime}$ end of the sense

180 strands results in $15-n t$ products, the preference for the $5^{\prime}$ phosphate can be quantitated

181 by comparing the proportion of 15-nt bands generated from TriP and MonoP substrates

182 (Figure 4). We found that OsDCL5 generated a lower proportion of 15-nt product from

183 TriP compared to MonoP substrates (Figure 4D), indicating that the $5^{\prime}$ triphosphate group

184 strongly inhibits OsDCL5-mediated cleavage. Similarly, the 5' triphosphate also

185 negatively affected dicing by AtDCL3, although the effect was less obvious than that on

186 OsDCL5 (Figure 4B). In contrast, the proportion of 15-nt products cleaved by OsDCL3

187 was similar for MonoP and TriP substrates (Figure 4C). Thus, the 5' triphosphate does

188 not affect OsDCL3-mediated dsRNA cleavage. In conclusion, DCL5 and DCL3 proteins

189 have different cleavage efficiencies based on the triphosphate group at the $5^{\prime}$ end of

190 dsRNA, likely impacting the small RNA substrates and pathways they can act upon in

191 plants. 
193 The PAZ domain determines DCL cleavage preferences based on the dsRNA 3'

194 structure.

195 Previous studies showed that the PAZ domain of Dicer proteins determines recognition

196 of the $3^{\prime}$ dsRNA structure, in humans and Drosophila $37,38,44,45$. The strong preference of

197 DCL3 for the $3^{\prime}$ overhang prompted us to hypothesize that the interaction between the

198 PAZ domain and the $3^{\prime}$ end of dsRNAs is required for DCL3-mediated dsRNA cleavage.

199 To test this, we introduced an extra phosphate group at the $3^{\prime}$ end of the antisense strand

200 of the lovr substrate (lovr 3' p) (Figure 5A). This modification is expected to sterically

201 block accommodation of the 3' overhang by the PAZ domain (Supplementary Figure 2).

202 If the $3^{\prime}$ phosphate inhibits substrate binding to the PAZ domain of DCLs, 24-nt fragments,

203 i.e. cleavage products from the $5^{\prime}$ end of the sense strand, should decrease. In contrast,

204 15-nt fragments, which represent cleavage from the $5^{\prime}$ end of antisense strand, should

205 increase. Our in vitro dicing assays with AtDCL3 or OsDCL3 showed a drastic decrease

206 in the 24-nt fragment and increase in the 15-nt fragment when the lovr $3^{\prime} \mathrm{p}$ substrate is

207 cleaved. This indicates that $3^{\prime}$ end recognition is important for dicing by AtDCL3 and

208 OsDCL3 (Figure 5B, C). In contrast, the proportion of 24-nt and 15-nt products

209 generated by OsDCL5 was not greatly altrered by the addition of a $3^{\prime}$ phosphate to the

210 antisense strand (Figure 5D). These data argue that the $3^{\prime}$ end of dsRNA is not strictly

211 recognized by the PAZ domain of DCL5.

212 To further confirm the importance of the PAZ domain for $3^{\prime}$ recognition, we

213 swapped the PAZ domains between OsDCL3 and OsDCL5 (Figure 6A). We named these 
214 chimeric proteins OsDCL3_PAZ5 and OsDCL5_PAZ3, and performed in vitro dicing

215 assays to investigate their preferences for 3' structures and 5' phosphate. As with OsDCL5,

216 we found that OsDCL3_PAZ5 prefers BLT substrates as well as substrates with 3'

217 overhangs (Figure 2D and 6B). In contrast, OsDCL5_PAZ3 showed a slight preference

218 for substrates with 3' overhangs, like OsDCL3 (Figure 2C and 6C). In addition, we

219 observed that OsDCL3_PAZ5 cleaved BLT substrates as accurately as OsDCL5 (Figure

220 2D and 6B), whereas OsDCL5_PAZ3 produced multiple bands, like OsDCL3 (Figure

221 2C and 6C). We also performed dicing assays using substrates with or without an extra

$2223^{\prime}$ phosphate on the antisense strand (lovr 3'p vs. 1ovr). Swapping PAZ domains between

223 OsDCL3 and OsDCL5 reversed the effects of blocking $3^{\prime}$ end recognition on each protein

224 (Supplementary Figure 3). Taken together, we conclude that the PAZ domain plays an

225 important role in determining the preference for $3^{\prime}$ structure and cleavage fidelity of

226 substrates with blunt ends.

227

228

The PAZ domain in DCL5 and DCL3 proteins determines 5' phosphate preference

229

on dsRNA substrates.

230 Previous studies have demonstrated that, in human Dicer, several basic amino acid residues in the "core" of the PAZ domain and its upstream Platform domain form a

232 binding pocket for the $5^{\prime}$ end of the dsRNA substrate ${ }^{38,43}$. Although the exact positions of

233 these residues are not conserved in Drosophila Dcr-2, alternative basic residues within

234 and upstream of the core PAZ domain are thought to form the binding pocket for the $5^{\prime}$

235 phosphorylated end of dsRNAs ${ }^{36}$. Since DCL3 and DCL5 lack corresponding basic 
236 residues upstream of the core PAZ domain (Supplementary Figure 4A), it remains

237 unclear which domain(s) discriminate the $5^{\prime}$ monophosphate from the $5^{\prime}$ triphosphate of

238 dsRNAs. Strikingly, we discovered that swapping the PAZ domains between OsDCL3

239 and OsDCL5 precisely altered 5' phosphate preference. OsDCL3_PAZ5 produced less

240 15-nt fragments from TriP than from MonoP (Figure 6D), indicating that OsDCL3_PAZ5

241 mimics OsDCL5 and prefers a 5' monophosphate. In contrast, OsDCL5_PAZ3 produced

242 similar amounts of 15-nt fragments from the MonoP and TriP substrates (Figure 6E),

243 indicating that, like OsDCL3, OsDCL5_PAZ3 does not discriminate 5' monophosphate

244 from triphosphate. Taken together, swapping the PAZ domain alters the preference for $5^{\prime}$

245 triphosphate in DCL5 and DCL3 proteins. These results suggest that, in addition to the 3'

246 structure, the PAZ domains of DCL3 and DCL5 determine 5' phosphate preference during

247 dsRNA cleavage. The PAZ domain therefore plays a key role in determining substrate

248 preference in plant small RNA-mediated silencing pathways.

\section{$250 \quad$ Discussion}

251 Role of PAZ domains in determining DCL3 family substrate preferences.

252 Previous studies have proposed that substrate preferences of human Dicer and Drosophila

253 Dcr-2 proteins are determined by the PAZ domain and helicase domain $36,38,39,43,46$. In our

254 study, swapping the PAZ domain was sufficient to alter substrate preferences for both 3'

255 structures and 5' triphosphates for OsDCL3 and OsDCL5. Our data demonstrate that the

256 PAZ domain alone can determine which dsRNA ends are preferred in DCL3 family

257 proteins. By comparing the amino acid sequences of PAZ domains across DCL3 family 
258 proteins, we identified a variable region where monocot DCL3 and DCL differ

259 (Supplementary Figure 4B). The corresponding region of human Dicer is located

260 between the $5^{\prime}$ and the $3^{\prime}$ binding pockets in the platform-PAZ cassette. The cassette forms

261 two structurally distinct complexes with short dsRNAs ${ }^{38}$; one has a visible $\alpha$-helix that

262 separates the two pockets, with the $3^{\prime}$ end of the dsRNA anchored in the $3^{\prime}$ pocket and the

2635 ' end released from the 5' pocket (Supplementary Figure 4C); the other has a disrupted

$264 \alpha$-helix that allows anchoring of both ends of the dsRNA in the two pockets. Thus, the $\alpha-$

265 helix is directly linked to substrate dsRNA binding. Given that the amino acid sequences

266 corresponding to the $\alpha$-helix in human Dicer differ significantly between monocot DCL3

267 and DCL5 (Supplementary Figure 4B), these sequences are expected to form distinct 5'

268 and $3^{\prime}$ binding pockets, which may impact dsRNA recognition in monocot DCL3 and

269 DCL5. Future structural analysis should reveal the exact mechanism by which the PAZ

270 domains of DCL3 and DCL5 recognize different substrates.

271

272 An additional rule for the biased processing of 24-nt heterochromatic siRNAs by

273 DCL3

274 Pol IV collaborates with RDR2 to generate 37-nt long dsRNA precursors, P4R2 RNAs,

275 for hc-siRNA production ${ }^{17}$. Interestingly, most of the sequenced mature hc-siRNAs are

276 produced from the $5^{\prime}$ end of the Pol IV strand ${ }^{17}$. Currently, this bias is thought to be

277 established by the preference for a $5^{\prime}$ adenine at the three different steps in the hc-siRNA

278 biogenesis: (1) start site selection by $\mathrm{Pol} \mathrm{IV}{ }^{17}$, (2) determination of the dicing direction

279 by DCL $3^{19}$, and (3) guide strand selection by AGO4/6 $6^{47}$. In addition to the $5^{\prime} \mathrm{A}$ preference, 
280 we found that the recognition of the $3^{\prime}$ end structure of the dsRNA precursor by DCL3 is

281 important for the establishment of substrate bias. A recent study showed that the precursor

282 dsRNA for hc-siRNAs has an asymmetric structure, with a 1-nt overhang at one end and

283 a blunt structure at the other end ${ }^{16}$. Our study with recombinant proteins and a previous

284 study with a crude cytoplasmic extract demonstrated that DCL3 prefers $3^{\prime}$ overhangs to

285 blunt-ended structures (Figure 2B, 2C) ${ }^{19}$. This substrate specificity will allow DCL3 to

286 preferentially cleave the dsRNA precursor from the 5' end of the Pol IV strand. This strand

287 forms a 1-nt 3' overhang structure with the 3' end of the RDR2 strand (Supplementary

288 Figure 5A, left) ${ }^{16}$. Cleavage from the opposite end would be less efficient because of its

289 blunt-ended nature (Supplementary Figure 5A, left) ${ }^{16}$. This $3^{\prime}$ structure bias would

290 further promote the biased production of $5^{\prime}$ adenine 24-nt siRNAs together with the $5^{\prime} \mathrm{A}$

291 preferences by Pol IV and DCL3. These multi-layer selection steps may facilitate the

292 formation of AGO4/6-RISC, resulting in efficient RNA-directed DNA methylation.

293

294 Preference for a 5' monophosphate in OsDCL5 determines the direction of cleavage

295 for phased 24-nt siRNA production

296 Specific miRNAs, including miR390 and 22-nt small RNAs, recruit RDR6 to the target

297 RNA to generate dsRNA precursors ${ }^{29}$. This long dsRNA is then processed into

298 phasiRNAs by DCLs ${ }^{28}$. Interestingly, DCLs always cleave precursors from the miRNA-

299 mediated cleavage site toward the other end. Although this fixed orientation of dicing is

300 important for production of functional phasiRNAs, how this is achieved has remained

301 unclear. In this study, we found that DCL5, which is known to produce phasiRNAs from 
302 RNAs with 22-nt miR2275 target sites, cleaves dsRNAs from a 5' monophosphate much

303 more efficiently than a $5^{\prime}$ triphosphate. Since the miRNA-cleaved end has a $5^{\prime}$

304 monophosphate, whereas the $5^{\prime}$ end of the RDR6 strand has a triphosphate in theory,

305 DCL5 substrate preference explains the directionality of dicing (Supplementary Figure

306 5A, right).

307 Although Arabidopsis does not produce 24-nt phasiRNAs, some eudicots do so

308 even without encoding DCL5 ${ }^{22,28}$. In these plants, DCL3 is likely to be responsible for

309 generating 24-nt phasiRNAs. We envision that the slight preference of eudicot DCL3 for

3105 ' monophosphorylated ends may also contribute to directional processing to produce

311 functional phasiRNA precursors.

312

313 The preference for $5^{\prime}$ triphosphates in OsDCL3 and AtDCL3 may enhance the

\section{4 production of heterochromatic siRNAs}

315 Since Pol IV uses nucleoside triphosphates (NTPs) as substrates for transcription and

316 lacks binding regions for the capping complexes, Pol IV-synthesized transcripts are

317 expected to possess a triphosphate group at the $5^{\prime}$ end ${ }^{15,21}$. RDR2 also generates $5^{\prime}$

318 triphosphate RNAs in vitro ${ }^{15,16}$. Thus, nascent P4R2 RNAs theoretically possess 5'

319 triphosphates at both ends. However, previous studies showed that the P4R2 RNAs that

320 accumulate in the $d c l 2 / 3 / 4$ mutant have monophosphates at the $5^{\prime}$ ends ${ }^{17,18,20}$, raising the

321 possibility that unknown RNA phosphatases convert the $5^{\prime}$ triphosphates of dsRNAs into

322 monophosphates in nuclei. In wild-type plants, DCL3 may encounter the P4R2 RNAs

323 before or after the tri- to monophosphate conversion. In any case, OsDCL3's ability to 
324 cleave both $5^{\prime}$ mono- and triphosphorylated dsRNAs with the same efficiency will

325 maximize the production of 24-nt heterochromatic siRNAs (Figure 5C and

326 Supplementary 5B). Given that AtDCL3 has a slight preference for 5'

327 monophosphorylated over triphosphorylated precursors (Figure 5B), dephosphorylation

328 prior to dicing may enhance the production of heterochromatic 24-nt siRNAs in

329 Arabidopsis thaliana.

330

331 Functional specialization of duplicated DCL3 genes in monocots

332 It is now believed that the appearance of DCL5 in monocots is explained by the "sub-

333 functionalization" of the ancestral DCL3 gene, which is speculated to function in the

334 production of both hc-siRNAs and phasiRNAs ${ }^{28}$. However, there is no biochemical

335 evidence supporting this hypothesis. One of the most interesting results in our study may

336 be that monocot OsDCL3 and OsDCL5 have completely different substrate specificities,

337 whereas eudicot AtDCL3 has an intermediate preference for dsRNAs with a $5^{\prime}$

338 triphosphate and $3^{\prime}$ overhang structure. This implies that monocot DCL5 and DCL3 were

339 not only subfunctionalized, but further optimized for cognate substrates after the

340 duplication from the ancient "eudicot-type" DCL3 (Figure 7). This functional

341 specialization process appears to have been achieved through accumulation of mutations

342 in the PAZ domain. Further biochemical studies on DCLs in a wider variety of plant

343 species will reinforce this hypothesis. Our data, however, indicate how OsDCL family

344 members have evolved to function in specific biological pathways. 


\section{$346 \quad$ Materials and Methods}

\section{$348 \quad$ Plasmid construction}

349 The primers used in this study are listed in Supplementary Table S1.

350 pASW-AtDCL3

351 AtDCL3 ORF was amplified from Arabidopsis thaliana (Col-0) cDNA using Oligo No.

3521 and 2 , and cloned into TOPO $^{\circledR}$ vector using pENTR $^{\mathrm{TM}} / \mathrm{D}-\mathrm{TOPO}^{\mathrm{TM}}$ Cloning Kit

353 (K240020). DCL3 fragment was then introduced into pASW vector using Gateway ${ }^{\mathrm{TM}}$ LR

354 Clonase $^{\mathrm{TM}}$ II Enzyme mix (Invitrogen ${ }^{\mathrm{TM}}$ 11791021). We noticed a missing $30 \mathrm{bp}$ in the

355 DCL3 sequence compared to the AT3G43920.2 reference sequence

356 (https://www.arabidopsis.org/servlets/TairObject?type=sequence\&id=4504453036). The

357 missing part was corrected by PCR using Oligo No. 3 and 4.

pASW-OsDCL3

360 OsDCL3a gene was cloned from the plasmid provided by National Agricultural and Food

361 Research Organization (Clone ID J013008L07), using Oligo No. 5 and 6 and then 362 assembled into pASW vector by NEBuilder Hifi DNA Assembly kit (NEB).

364 pASW-OsDCL5

365 Four DNA fragments of the OsDCL5 ORF (199-927 nt, 912-2478 nt, 2389-4785 nt and

366 4786-4914 nt) were amplified from Oryza Sativa anther cDNA using Oligos No. 7-14.

367 A DNA fragment corresponding to 1-198 nt of OsDCL5 ORF could not be amplified 
368 from anther cDNA, and was therefore synthesized by PCR using Oligos No. 15-19

369 according to the reference sequence of OsDCL3b (CDS) from rap-db (The Rice

370 Annotation

Project

Database)

371 (https://rapdb.dna.affrc.go.jp/viewer/gene_detail/irgsp1?name=Os10t0485600-

372 01;feature id=339409). These five DNA fragments (1-198 nt, 199-927 nt, 912-2478 nt,

$373 \quad 2389-4785 \mathrm{nt}$ and $4786-4914 \mathrm{nt}$ ) were assembled into the pASW vector using NEBuilder

374 Hifi DNA Assembly kit (NEB).

375

$376 \quad$ PAZ exchange plasmids

377 DNA fragments comprising 2560-3030 nt of OsDCL3 and OsDCL5 ORFs were

378 exchanged between the two genes. The plasmids (pASW-OsDCL3_PAZ5 and pASW-

379 OsDCL5_PAZ3) were prepared by PCR using Oligos No. 20-27 and the NEBuilder Hifi

380 DNA Assembly kit (NEB).

381

382

383 Cell culture

384 Drosophila Schneider 2 cells (S2 cells) were cultured in Schneider's Drosophila Medium

385 (Gibco) supplemented with 10\% (v/v) Fetal Bovine Serum (FBS) (Sigma) and antibiotics

386 at $28^{\circ} \mathrm{C}$, sealed with parafilm.

387

388 Production of SBP-tagged AtDCL3, OsDCL3, OsDCL5 proteins in Drosophila S2

389

cells 
390 S2 cells $\left(1-1.5 \times 10^{7}\right.$ cells $/ 10 \mathrm{~cm}$ dish $)$ were transfected with $10 \mu \mathrm{g}$ pASW plasmids carrying plant DCL3 family genes (pASW-AtDCL3, OsDCL3 or OsDCL5) with $20 \mu 1$

392 X-tremeGENETM HP DNA Transfection Reagent (Roche) following manufacturer's instructions. The transfected cells were harvested after 72 hours for lysate preparation.

\section{Cell lysate preparation}

396 S2 cells were harvested by centrifugation using a swinging-bucket rotor at $1,500 \times \mathrm{g}$ for

$3973 \mathrm{~min}$ at room temperature. The cell pellet was washed by cold PBS (pH 7.4) and was centrifuged at $1,500 \times g$ at $4^{\circ} \mathrm{C}$. The pellets were then weighed and resuspended in equal volumes of Hypotonic buffer [10 mM HEPES-KOH (pH 7.4), $10 \mathrm{mM}$ potassium acetate, supernatant was flash frozen in liquid nitrogen and immediately stored at $-80^{\circ} \mathrm{C}$ in singleuse aliquots.

\section{Protein purification by Streptavidin beads}

409 Streptavidin Sepharose High Performance beads (GE Healthcare), equivalent to $25 \%$ of

410 the lysate by volume, were washed with $1 \mathrm{ml}$ lysis buffer [30 mM HEPES-KOH (pH 7.4),

$411100 \mathrm{mM}$ potassium acetate, $2 \mathrm{mM}$ magnesium acetate], and then mixed gently with the 
412 lysate. The suspension was incubated for 1 hour at $4^{\circ} \mathrm{C}$ on a rotator and then washed three

413 times with wash buffer $(1 \times$ lysis buffer containing $800 \mathrm{mM} \mathrm{NaCl}$ and $1 \%(\mathrm{v} / \mathrm{v})$ Triton $\mathrm{X}$ -

414 100). The SBP-tagged protein was then eluted with biotin elution buffer $(1 \times$ lysis buffer,

$4155 \mathrm{mM}$ DTT, $30 \%$ glycerol and $2.5 \mathrm{mM}$ biotin) at $4{ }^{\circ} \mathrm{C}$ on a rotator for 20 minutes, and the

416 elution step repeated 3 times. The eluates were flash frozen in liquid nitrogen and

417 immediately stored at $-80^{\circ} \mathrm{C}$ in single-use aliquots after adding BSA to a final

418 concentration of $0.2 \mathrm{mg} / \mathrm{ml}$.

\section{Preparation of radiolabeled dsRNA substrates}

421 The sequences of the sense and antisense RNAs used in this study are following:

38-nt sense strand, GAAUUGCUCAACAGUAUGGGCAUUUGACGCAGCCUCCC;

blunt (antisense), GGGAGGCUGCGUCAAAUGCCCAUACUGUUGAGCAAUUC;

lovr (antisense), GGAGGCUGCGUCAAAUGCCCAUACUGUUGAGCAAUUCC;

2ovr (antisense), GAGGCUGCGUCAAAUGCCCAUACUGUUGAGCAAUUCAC.

426 Single-stranded RNAs with a 5' hydroxyl group (OH) were synthesized by GeneDesign

427 Inc.(Osaka Japan), while the sense strand RNA with a 5' triphosphate was synthesized by

428 Bio-Synthesis (Texas, USA). The antisense strand with a $3^{\prime}$ phosphate was radiolabeled

429 by T4 Polynucleotide Kinase (3' phosphatase minus) (NEB) and $\left[\gamma-{ }^{32} \mathrm{P}\right] \mathrm{ATP}$. Strands with

430 a $5^{\prime}$ monophosphate were radiolabeled with T4 polynucleotide kinase (Takara) and $[\gamma-$

$\left.431{ }^{32} \mathrm{P}\right]$ ATP. The sense and antisense strands were heat-annealed in lysis buffer as previously

432 described ${ }^{48}$. The annealed dsRNAs were then separated by electrophoresis on $15 \%$ native

433 polyacrylamide gels. The dsRNAs in gel pieces were excised and eluted by soaking in 2 
$434 \times$ elution buffer [200 mM Tris- $\mathrm{HCl}(\mathrm{pH} 7.5), 2 \mathrm{mM} \mathrm{MgCl} 2,300 \mathrm{mM} \mathrm{NaCl}, 2 \% \mathrm{SDS}]$

435 overnight at room temperature. dsRNAs were mixed with glycogen and precipitated by

436 isopropanol, then dissolved in lysis buffer.

437

438 Dicing assay

439 Three nanomolar ${ }^{32} \mathrm{P}-$ labeled dsRNAs and 1 or $2 \mathrm{nM}$ purified recombinant proteins were

440 incubated in $1 \times$ lysis buffer containing $5 \mathrm{mM}$ DTT, $5 \mathrm{mM}$ magnesium acetate, ATP

441 regeneration system $[25 \mathrm{mM}$ creatine phosphate (Sigma), $1 \mathrm{mM} \mathrm{ATP,} 0.03 \mathrm{U} / \mu 1$ creatin

442 kinase (Calbiochem)], and $0.1 \mathrm{U} / \mu 1 \mathrm{RNasin}$ (Promega) at $25^{\circ} \mathrm{C}$. To draw time course

443 curves for each reaction, $2 \mu 1$ of the reaction mixture was taken at 5,10, 20, 30, 45 and 60

444 minutes after the reaction was started, were mixed with $8 \mu 1$ of low-salt PK solution

$445[0.125 \%$ SDS, 12.5 mM EDTA, 12.5 mM HEPES-KOH (pH 7.4), 12.5\% Proteinase K],

446 and then incubated at $50^{\circ} \mathrm{C}$ for $10 \mathrm{~min}$. An equal volume of $2 \times$ formamide dye $[10 \mathrm{mM}$

447 EDTA ( $\mathrm{pH} 8.0), 98 \%(\mathrm{w} / \mathrm{v})$ deionized formamide, $0.025 \%(\mathrm{w} / \mathrm{v})$ xylene cyanol, $0.025 \%$

448 bromophenol blue] was then added and incubated at $95^{\circ} \mathrm{C}$ for $2 \mathrm{~min}$. The cleavage

449 products were analyzed on $15 \%$ denaturing polyacrylamide gels and detected by

450 autoradiography. Small RNA products were quantitated from relative band intensities

451 measured with a Typhoon FLA 7000 image analyzer (GE Healthcare Life Sciences) and

452 quantified using MultiGauge software (Fujifilm Life Sciences). Graphs were prepared

453 using GraphPad Prism 8. 


\section{Author Contributions}

456 S.C. and H.-o.I. designed the project. S.C and W.L. performed biochemical experiments.

457 Y.T. and H.-o.I. supervised the project. S.C. N.M, Y.T. and H.-o.I. analyzed data. S.C., 458 Y.T. and H.-o.I. wrote the manuscript with edits provided by all authors. All authors

459 discussed the results and approved the manuscript.

460

\section{$461 \quad \underline{\text { Acknowledgements }}$}

462 We thank Reina Komiya for providing total RNA from the anther of Oryza Sativa. We

463 also thank all the members of the Tomari laboratory for discussion and critical comments

464 on the manuscript, and Life Science Editors for editorial assistance. S.C. gratefully 465 acknowledges the China Scholarship Council (CSC, No. 201808050036). This work was

466 supported by JST, PRESTO (grant JPMJPR18K2 to H.-o.I).

467 


\section{$468 \quad$ Figure legends}

$469 \quad$ Figure 1. In vitro assay determines substrate specificities of DCL3 and DCL5.

470 (A) Schematic illustrating in vivo substrates for DCL3 and DCL5. The dsRNA substrates

471 for DCL3, named Pol IV and RDR2-dependent RNAs (P4R2 RNAs), have 5'

472 triphosphates on both strands in theory, which might be converted to monophosphates

473 before cleavage by DCL3. The $3^{\prime}$ end structures of the two strands of P4R2 RNAs are

474 different, and carry a 1-nt overhang (1-nt ovr) on one strand (RDR2 strand) and blunt end

475 (BLT) on the other strand (Pol IV strand). The cleavage direction is shown with a blue

476 arrow. DCL5 substrates are miR2275 targets, with dsRNA generated by RDR6. Such

477 phasiRNA precursors generally carry heterogenous 3' structures. The 5' phosphorylation

478 status of phasiRNA precursors is different on the two strands: with a $5^{\prime}$ monophosphate

479 on one strand (miRNA target strand) and a $5^{\prime}$ triphosphate on the other strand (RDR6

480 strand). The cleavage direction is shown with a pink arrow.

481 (B) Coomassie brilliant blue staining of recombinant AtDCL3, OsDCL3 and OsDCL5 482 proteins.

483 (C) Schematic of in vitro dicing assays. dsRNA substrates can be cleaved from the 5' ends 484 of both strands, mainly generating two cleaved fragments: $24-$ nt and 14-16-nt. The 485 products of dicing assays were analyzed by denaturing gel. Band A, B and C represent 486 full-length RNA, 24-nt and 14-16-nt products, respectively. Cleavage ratio is calculated 487 by dividing the sum of the cleaved fragments $(B+C)$ by the total amounts of the substrate $488 \quad(\mathrm{~A}+\mathrm{B}+\mathrm{C})$. 
491 (A) 38-nt substrates radiolabeled on the $5^{\prime}$ monophosphate of sense strands, with different

492 3' structures: blunt (BLT), 3' 1-nt overhang (1 ovr) and 3' 2-nt overhang (2 ovr), were 493 used in the dicing assays. Red arrowheads and grey arrowheads indicate cleavage from 494 the $5^{\prime}$ end of sense strands and antisense strands, respectively. These substrates can be 495 cleaved into 24-nt (band B) and 14,15 or 16-nt (band C) products according to their 3' 496 structures. Cleavage efficiency is calculated using $(B+C) /(A+B+C)$.

497 (B, C, D) Left panel: Representative gel images of dicing assays by (B) AtDCL3, (C) 498 OsDCL3 and (D) OsDCL5 cleaving the 38-nt substrates with different 3' structures (from 499 left to right: BLT, 1ovr and 2ovr). The double and triple asterisks indicate the inaccurate 500 cleavage products, long and short, respectively. Right panel: Quantification of cleavage 501 efficiency in the left panel. For BLT, bands of inaccurate cleavage products (long and 502 short fragments) were also calculated as cleavage products. The mean values \pm SD from 503 three independent experiments are shown. AtDCL3 and OsDCL3 both prefer substrates 504 with 3' overhangs. OsDCL5 does not show preferences for specific 3' structures.

506 Figure 3. The 5' monophosphate is important for catalysis by DCL3 and DCL5.

507 (A) 1-nt 3' overhang substrates radiolabeled on the 5' monophosphate of antisense strands, 508 with a $5^{\prime}$ hydroxyl groups $(\mathrm{OH})$ or a monophosphate (MonoP) on sense strands, were used 509 for dicing assays. Red arrowheads and grey arrowheads indicate cleavage from the 5' end 510 of sense strands and antisense strands, respectively. Cleavage from the $5^{\prime}$ end of sense 
511 strands results in 15-nt products (band C), and the proportion of cleavage from the $5^{\prime}$ end

512 of sense strands is calculated by $\mathrm{C} /(\mathrm{A}+\mathrm{B}+\mathrm{C})$.

513 (B, C, D) Left panel: Representative gel images of dicing assays by (B) AtDCL3, (C)

514 OsDCL3 and (D) OsDCL5 cleaving 1-nt overhang MonoP and OH substrates. Right

515 panel: Quantification of cleavage from the 5' end of sense strands (15-nt bands) in the left

516 panel. The mean values \pm SD from three independent experiments are shown. Compared

517 with a $5^{\prime}$ monophosphate, a 5' hydroxyl group greatly reduced cleavage from the $5^{\prime}$ end

518 of sense strand by AtDCL3, OsDCL3 and OsDCL5.

520 Figure 4. DCL3 and DCL5 have different preferences for the 5' triphosphate

521 (A) Dicing assays were conducted using 1-nt 3' overhang substrates radiolabeled on the

523 triphosphate (TriP) on the sense strands. Red arrowheads and grey arrowheads indicate

524 cleavage from the $5^{\prime}$ end of sense strands and antisense strands, respectively. Cleavage

525 from the $5^{\prime}$ end of sense strands results in 15-nt products (band C), and the proportion of 526 cleavage from the $5^{\prime}$ end of sense strands is calculated as $\mathrm{C} /(\mathrm{A}+\mathrm{B}+\mathrm{C})$.

527 (B, C, D) Left panel: Representative gel image of dicing assays with (B) AtDCL3, (C)

528 OsDCL3 and (D) OsDCL5 cleaving MonoP and TriP substrates. Right panel:

529 Quantification of the proportion of cleavage from the $5^{\prime}$ end of sense strands (15-nt bands)

530 in the left panel. The mean values \pm SD from three independent experiments are shown.

531 AtDCL3 slightly prefers substrates with $5^{\prime}$ monophosphate. OsDCL3 does not show an 
532 obvious preference for the 5' mono- or triphosphate. OsDCL5 prefers substrates carrying

533 a 5' monophosphate.

Figure 5. Recognition of the 3' hydroxyl group is important for DCL3, but not DCL5,

cleavage

537 (A) Dicing assays were conducted on 1-nt 3' overhang substrates radiolabeled on the 5'

538 monophosphate of sense strands (1ovr). To disrupt recognition of the $3^{\prime}$ hydroxyl, an extra

539 monophosphate group was added to the $3^{\prime}$ end of the antisense strand (1ovr 3'p). Red

540 arrowheads and grey arrowheads indicate cleavage from the $5^{\prime}$ end of sense strands and

541 antisense strands, respectively. Cleavage from 5' end of sense strands (corresponding to

542 the $3^{\prime}$ phosphate on antisense strands) results in 24-nt products (band B).

543 (B, C, D) Left panel: Representative gel images of dicing assays by (B) AtDCL3, (C)

544 OsDCL3 and (D) OsDCL5 cleaving 1-nt overhang substrates with or without a 3'

545 monophosphate on the antisense strand (lovr 3'p or 1ovr). Right panel: Quantification of

546 the proportion of cleavage from the $5^{\prime}$ end of sense strands (24-nt bands) is shown in the

547 left panel. Mean values \pm SD from three independent experiments are shown. The extra

$5483^{\prime}$ phosphate greatly reduced cleavage from the $5^{\prime}$ end of sense strand by AtDCL3 and

549 OsDCL3 compared with the lovr substrate, but only moderately reduced cleavage from

550 the 5' end of sense strand by OsDCL5.

552 Figure 6. The PAZ domains plays an important role in specific DCL3 and DCL5 
554 (A) Swapping the OsDCL3 and OsDCL5 PAZ domains generates two chimeric proteins:

\section{OsDCL3_PAZ5 and OsDCL5_PAZ3.}

556 (B, C) Dicing assays by (B) OsDCL3_PAZ5 and (C) OsDCL5_PAZ3 with dsRNA

557 substrates holding different 3' structures. Left panel: Representative gel images of dicing 558 assays by OsDCL3_PAZ5 and OsDCL5_PAZ3 cleaving 38-nt substrates BLT, 1ovr and 559 2ovr. The double and triple asterisks indicate the inaccurate cleavage products, long and 560 short, respectively. Right panel: Quantification of dicing efficiency in the left panel. For 561 BLT, bands of inaccurate cleavage products (long and short fragments) were also 562 calculated as cleavage products. Mean values \pm SD from three independent experiments 563 are shown. OsDCL3_PAZ5 does not show an obvious preference for specific 3' structures, 564 while OsDCL5_PAZ3 prefers substrates with a 3' overhang rather than blunt ends.

565 (D, E) Dicing assays by (D) OsDCL3_PAZ5 and (E) OsDCL5_PAZ3 with MonoP and 566 TriP dsRNA substrates. Left panel: Representative gel images of dicing assays by 567 OsDCL3_PAZ5 and OsDCL5_PAZ3 cleaving 38-nt substrates, MonoP and TriP. Right 568 panel: Quantification of the proportion of cleavage from the $5^{\prime}$ end of sense strands (15569 nt bands) in the left panel. The mean values \pm SD from three independent experiments 570 are shown. OsDCL3_PAZ5 prefers 5' monophosphate, while OsDCL5_PAZ3 does not 571 discriminate 5' monophosphate from triphosphate.

573 Figure 7. Summary of functions and substrate preferences of DCL3/5 family 574 proteins

575 Summary of substrate functions and specificities of eudicot DCL3, monocot DCL3 and 
576 DCL5. Monocot OsDCL3 and OsDLC5 have completely distinct substrate specificities,

577 whereas eudicot AtDCL3 has an intermediate preference for dsRNAs. These data argue

578 that monocot DCL5 and DCL3 duplicated from a common ancestral "eudicot-type"

579 DCL3, then subfunctionalized and further optimized to cleave cognate substrates.

580 


\section{$\underline{\text { References }}$}

582 1. Voinnet, O. Origin, Biogenesis, and Activity of Plant MicroRNAs. Cell 136, 583 669-687 (2009).

$5842 . \quad$ Ding, S.-W. \& Voinnet, O. Antiviral Immunity Directed by Small RNAs. Cell 585 130, 413-426 (2007).

586 3. Bartel, D. P. Metazoan MicroRNAs. Cell 173, 20-51 (2018).

587 4. D’Ario, M., Griffiths-Jones, S. \& Kim, M. Small RNAs: Big Impact on Plant 588 Development. Trends in Plant Science (2017) doi:10.1016/j.tplants.2017.09.009.

589 5. Bernstein, E., Caudy, A. A., Hammond, S. M. \& Hannon, G. J. Role for a

590 bidentate ribonuclease in the initiation step of RNA interference. Nature (2001)

591 doi:10.1038/35053110.

592 6. Song, M. S. \& Rossi, J. J. Molecular mechanisms of Dicer: Endonuclease and 593 enzymatic activity. Biochemical Journal (2017) doi:10.1042/BCJ20160759.

$5947 . \quad$ Bologna, N. G. \& Voinnet, O. The Diversity, Biogenesis, and Activities of

595 Endogenous Silencing Small RNAs in Arabidopsis. Annual Review of Plant Biology 65, 596 473-503 (2014).

597 8. Wu, L. et al. DNA Methylation Mediated by a MicroRNA Pathway.

598 Molecular Cell 38, 465-475 (2010).

5999 9. Law, J. A. \& Jacobsen, S. E. Establishing, maintaining and modifying DNA

600 methylation patterns in plants and animals. Nature Reviews Genetics 11, 204-220

601 (2010).

602 10. Matzke, M. A. \& Mosher, R. A. RNA-directed DNA methylation: an

603 epigenetic pathway of increasing complexity. Nature Reviews Genetics 15, 394-408 604 (2014).

605 11. Erdmann, R. M. \& Picard, C. L. RNA-directed DNA Methylation. PLOS

606 Genetics 16, e1009034 (2020).

607 12. Xie, Z. et al. Genetic and functional diversification of small RNA pathways in 608 plants. PLoS Biology (2004) doi:10.1371/journal.pbio.0020104.

609 13. Herr, A. J., Jensen, M. B., Dalmay, T. \& Baulcombe, D. C. RNA polymerase 610 IV directs silencing of endogenous DNA. Science (2005) doi:10.1126/science.1106910.

611 14. Onodera, Y. et al. Plant nuclear RNA polymerase IV mediates siRNA and

612 DNA methylation- dependent heterochromatin formation. Cell (2005) 
613 doi:10.1016/j.cell.2005.02.007.

614 15. Haag, J. R. et al. In Vitro Transcription Activities of Pol IV, Pol V, and

615 RDR2 Reveal Coupling of Pol IV and RDR2 for dsRNA Synthesis in Plant RNA

616 Silencing. Molecular Cell (2012) doi:10.1016/j.molcel.2012.09.027.

617 16. Singh, J., Mishra, V., Wang, F., Huang, H. Y. \& Pikaard, C. S. Reaction

618 Mechanisms of Pol IV, RDR2, and DCL3 Drive RNA Channeling in the siRNA-

619 Directed DNA Methylation Pathway. Molecular Cell (2019)

620 doi:10.1016/j.molcel.2019.07.008.

621 17. Zhai, J. et al. A one precursor one siRNA model for pol IV-dependent siRNA

622 biogenesis. Cell (2015) doi:10.1016/j.cell.2015.09.032.

623 18. Blevins, T. et al. Identification of pol IV and RDR2-dependent precursors of

$62424 \mathrm{nt}$ siRNAs guiding de novo DNA methylation in arabidopsis. eLife (2015)

625 doi:10.7554/eLife.09591.

$62619 . \quad$ Nagano, H., Fukudome, A., Hiraguri, A., Moriyama, H. \& Fukuhara, T.

627 Distinct substrate specificities of Arabidopsis DCL3 and DCL4. Nucleic Acids Research

628 42, 1845-1856 (2014).

629 20. Li, S. et al. Detection of Pol IV/RDR2-dependent transcripts at the genomic

630 scale in Arabidopsis reveals features and regulation of siRNA biogenesis. Genome Res.

$63125,235-245$ (2015).

632 21. Wendte, J. M. \& Pikaard, C. S. The RNAs of RNA-directed DNA

633 methylation. Biochimica et Biophysica Acta (BBA) - Gene Regulatory Mechanisms

634 1860, 140-148 (2017).

635 22. Xia, R. et al. 24-nt reproductive phasiRNAs are broadly present in

636 angiosperms. Nature Communications (2019) doi:10.1038/s41467-019-08543-0.

637 23. Johnson, C. et al. Clusters and superclusters of phased small RNAs in the

638 developing inflorescence of rice. Genome Res 19, 1429-1440 (2009).

639 24. Zhai, J. et al. Spatiotemporally dynamic, cell-type-dependent premeiotic and

640 meiotic phasiRNAs in maize anthers. PNAS 112, 3146-3151 (2015).

$64125 . \quad$ Chen, H.-M. et al. 22-nucleotide RNAs trigger secondary siRNA biogenesis

642 in plants. PNAS 107, 15269-15274 (2010).

643 26. Cuperus, J. T. et al. Unique functionality of 22-nt miRNAs in triggering

644 RDR6-dependent siRNA biogenesis from target transcripts in Arabidopsis. Nat Struct

645 Mol Biol 17, 997-1003 (2010). 
646 27. Fei, Q., Xia, R. \& Meyers, B. C. Phased, Secondary, Small Interfering RNAs

647 in Posttranscriptional Regulatory Networks. The Plant Cell 25, 2400-2415 (2013).

648 28. Liu, Y., Teng, C., Xia, R. \& Meyers, B. C. PhasiRNAs in Plants: Their

649 Biogenesis, Genic Sources, and Roles in Stress Responses, Development, and

650 Reproduction. Plant Cell 32, 3059-3080 (2020).

651 29. Sakurai, Y. et al. Cell-free reconstitution reveals the molecular mechanisms

652 for the initiation of secondary siRNA biogenesis in plants. Proc Natl Acad Sci USA 118, 653 e2102889118 (2021).

$65430 . \quad$ Rajeswaran, R. et al. Sequencing of RDR6-dependent double-stranded RNAs

655 reveals novel features of plant siRNA biogenesis. Nucleic Acids Research (2012)

656 doi:10.1093/nar/gks242.

657 31. Curaba, J. \& Chen, X. Biochemical activities of arabidopsis RNA-dependent

658 RNA polymerase 6. Journal of Biological Chemistry (2008)

659 doi:10.1074/jbc.M708983200.

660 32. Baeg, K., Iwakawa, H. O. \& Tomari, Y. The poly(A) tail blocks RDR6 from

661 converting self mRNAs into substrates for gene silencing. Nature Plants (2017)

662 doi:10.1038/nplants.2017.36.

663 33. Song, X. et al. Roles of DCL4 and DCL3b in rice phased small RNA

664 biogenesis: Phased small RNA clusters biogenesis in rice. The Plant Journal 69, 462-

665474 (2012).

$66634 . \quad$ Svobodova, E., Kubikova, J. \& Svoboda, P. Production of small RNAs by

667 mammalian Dicer. Pflugers Arch - Eur J Physiol 468, 1089-1102 (2016).

668 35. Fukudome, A. \& Fukuhara, T. Plant dicer-like proteins: double-stranded

669 RNA-cleaving enzymes for small RNA biogenesis. J Plant Res 130, 33-44 (2017).

$67036 . \quad$ Kandasamy, S. K. \& Fukunaga, R. Phosphate-binding pocket in Dicer-2 PAZ

671 domain for high-fidelity siRNA production. Proc Natl Acad Sci USA 113, 14031-14036

672 (2016).

673 37. Ma, J.-B., Ye, K. \& Patel, D. J. Structural basis for overhang-specific small

674 interfering RNA recognition by the PAZ domain. Nature 429, 318-322 (2004).

675 38. Tian, Y. et al. A Phosphate-Binding Pocket within the Platform-PAZ-

676 Connector Helix Cassette of Human Dicer. Molecular Cell (2014)

677 doi:10.1016/j.molcel.2014.01.003.

$67839 . \quad$ Sinha, N. K., Iwasa, J., Shen, P. S. \& Bass, B. L. Dicer uses distinct modules 
679 for recognizing dsRNA termini. Science (2018) doi:10.1126/science.aaq0921.

680 40. Sinha, N. K., Trettin, K. D., Aruscavage, P. J. \& Bass, B. L. Drosophila dicer-

6812 cleavage is mediated by helicase- and dsRNA termini-dependent states that are

682 modulated by Loquacious-PD. Mol Cell 58, 406-417 (2015).

683 41. Welker, N. C. et al. Dicer's Helicase Domain Discriminates dsRNA Termini

684 to Promote an Altered Reaction Mode. Molecular Cell 41, 589-599 (2011).

685 42. Mukherjee, K., Campos, H. \& Kolaczkowski, B. Evolution of Animal and

686 Plant Dicers: Early Parallel Duplications and Recurrent Adaptation of Antiviral RNA

687 Binding in Plants. Molecular Biology and Evolution 30, 627-641 (2013).

688 43. Park, J. E. et al. Dicer recognizes the 5 ' end of RNA for efficient and accurate

689 processing. Nature (2011) doi:10.1038/nature10198.

690 44. Fukunaga, R., Colpan, C., Han, B. W. \& Zamore, P. D. Inorganic phosphate

691 blocks binding of pre-miRNA to Dicer-2 via its PAZ domain. EMBO Journal (2014)

692 doi:10.1002/embj.201387176.

693 45. Liu, Z. et al. Cryo-EM Structure of Human Dicer and Its Complexes with a

694 Pre-miRNA Substrate. Cell 173, 1191-1203.e12 (2018).

695 46. Naganuma, M., Tadakuma, H. \& Tomari, Y. Single-molecule analysis of

696 processive double-stranded RNA cleavage by Drosophila Dicer-2. Nat Commun 12,

6974268 (2021).

698 47. Mi, S. et al. Sorting of Small RNAs into Arabidopsis Argonaute Complexes

699 Is Directed by the 5' Terminal Nucleotide. Cell 133, 116-127 (2008).

700 48. Tomari, Y. \& Iwakawa, H.-O. In Vitro Analysis of ARGONAUTE-Mediated

701 Target Cleavage and Translational Repression in Plants. Methods Mol Biol 1640, 55-71

702 (2017).

703

704 


\section{Chen et al., Figure 1}

\section{A}
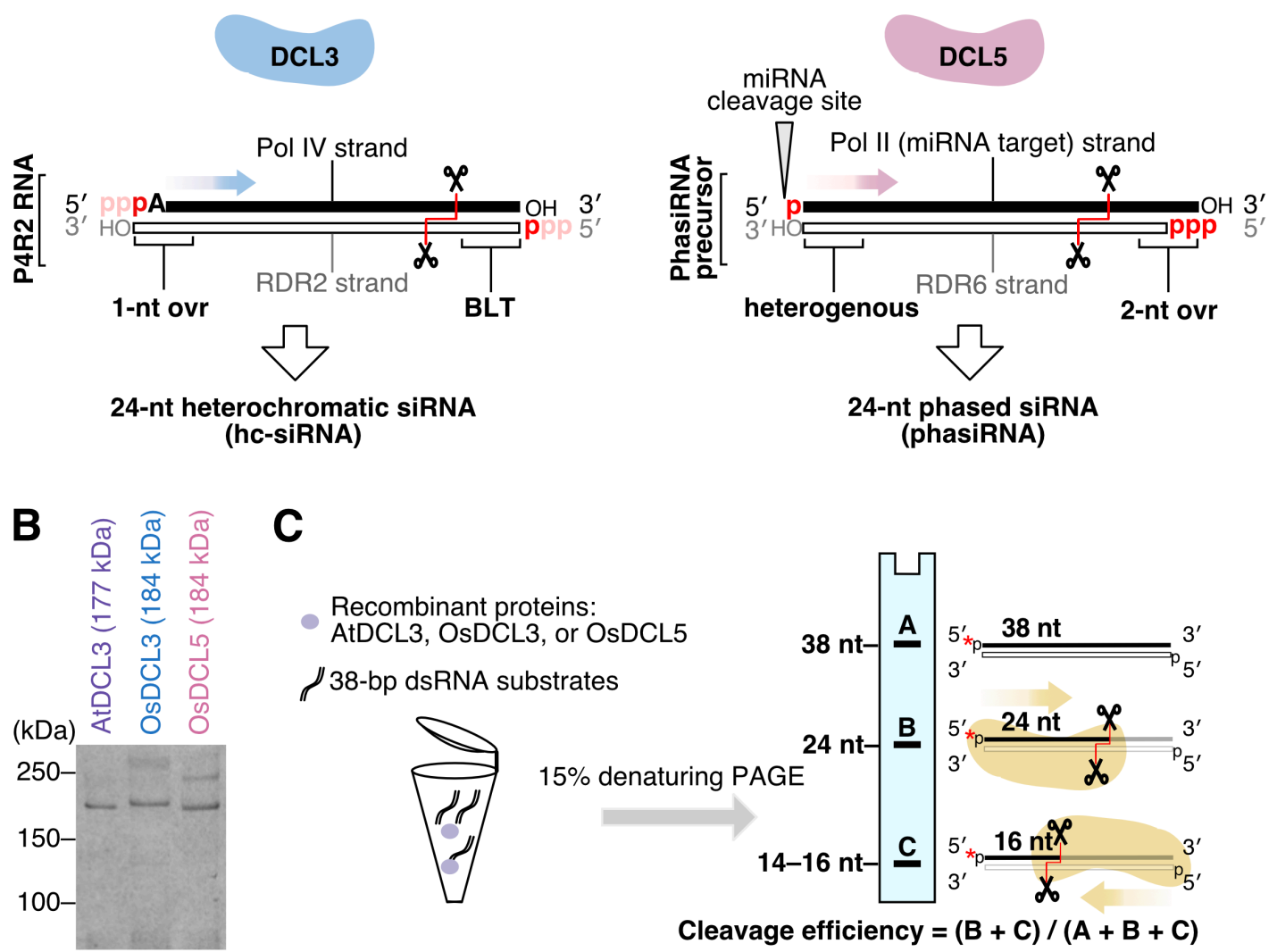

C

Recombinant proteins:

AtDCL3, OsDCL3, or OsDCL5

38-bp dsRNA substrates
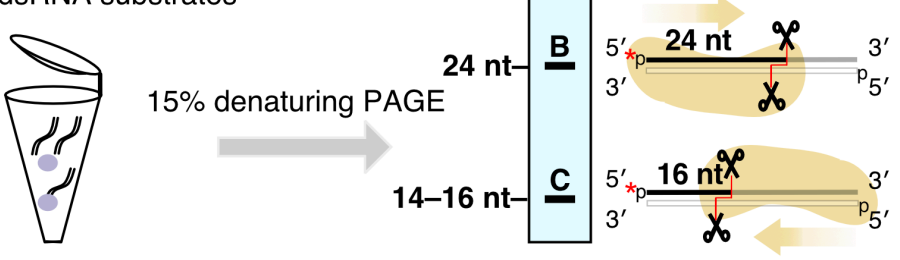

Cleavage efficiency $=(B+C) /(A+B+C)$ 


\section{Chen et al., Figure 2}

A

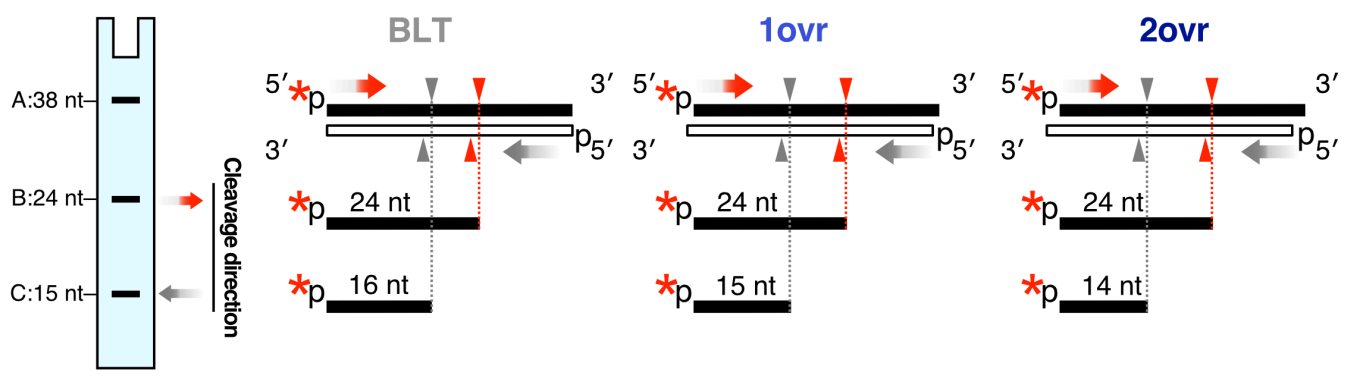

B (min) $\frac{\text { BLT }}{51020304560} \frac{\text { 1ovr }}{51020304560} \frac{\text { 2ovr }}{51020304560}$ $38 \mathrm{nt}-$ -

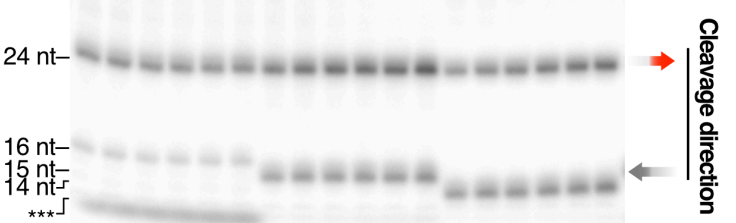

C

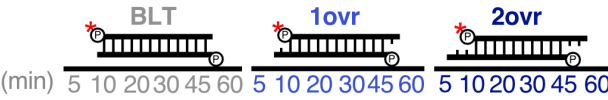

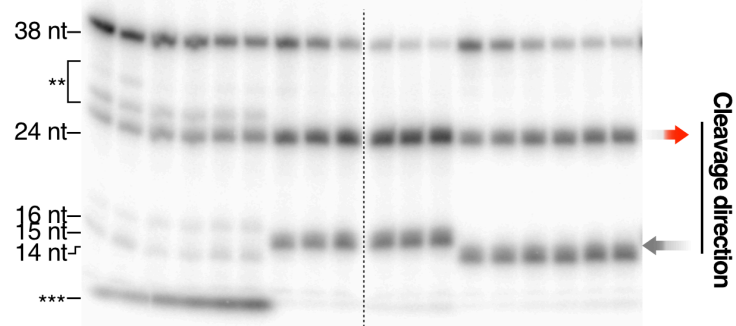

D
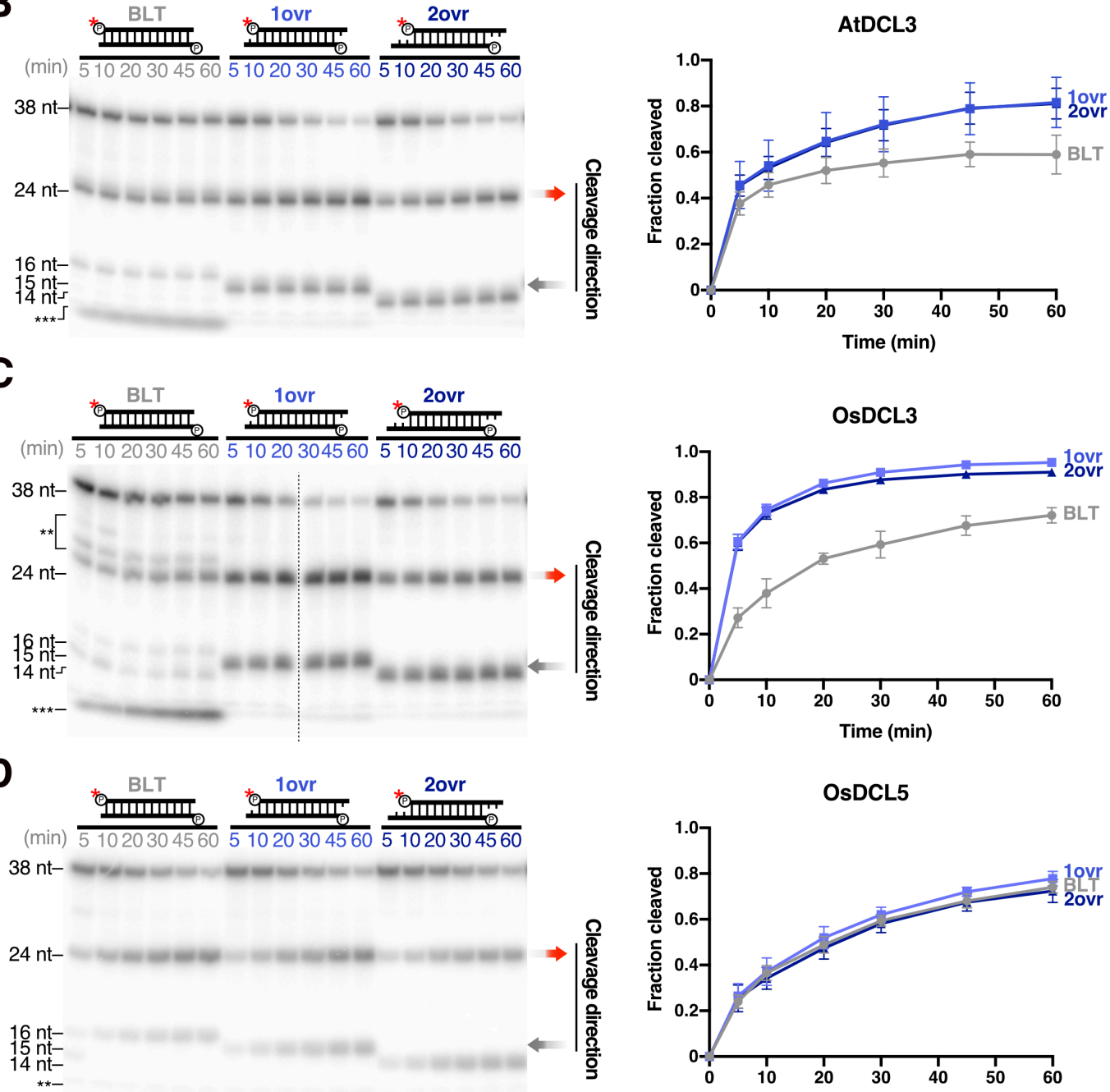

OsDCL5

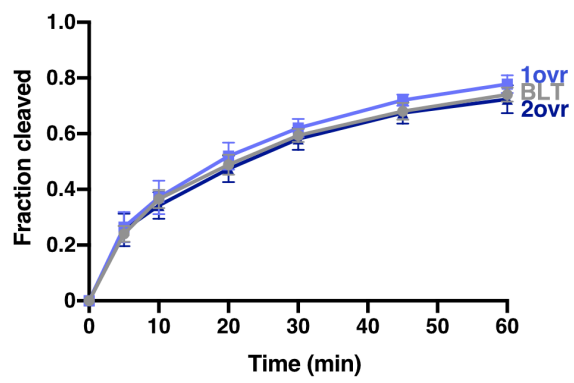


A

Chen et al., Figure 3

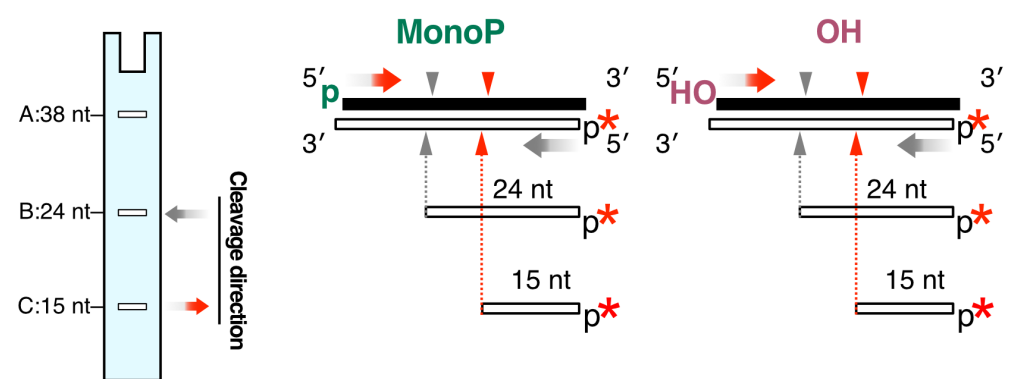

B
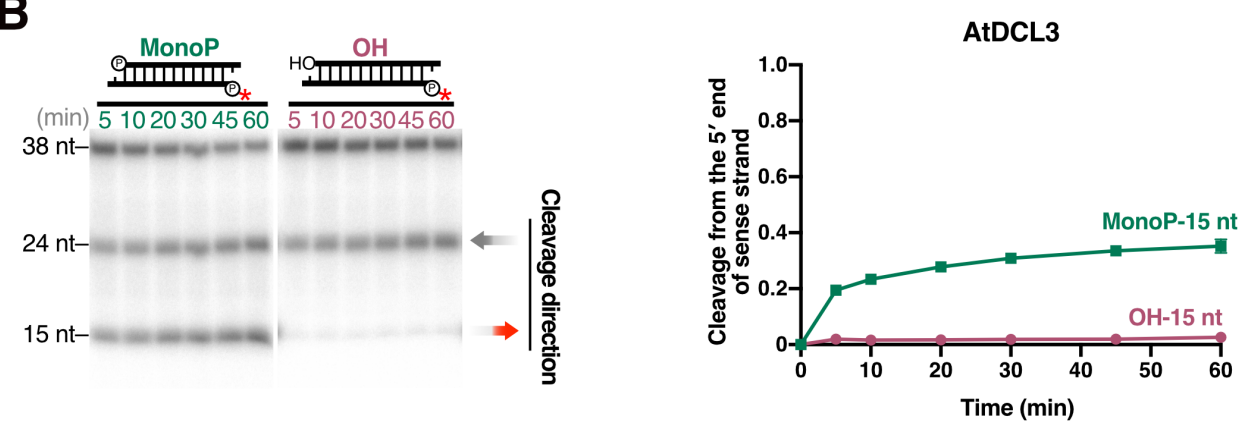

C
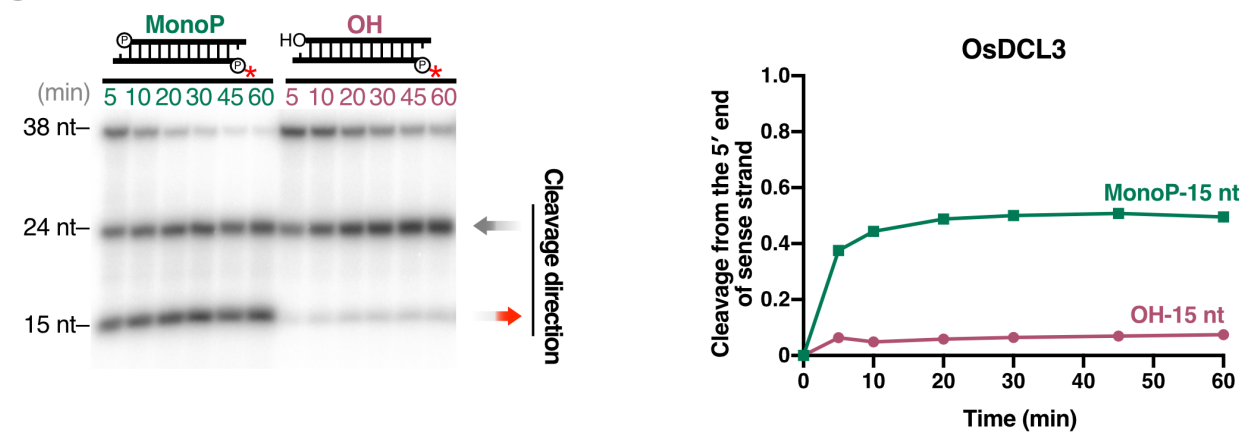

D
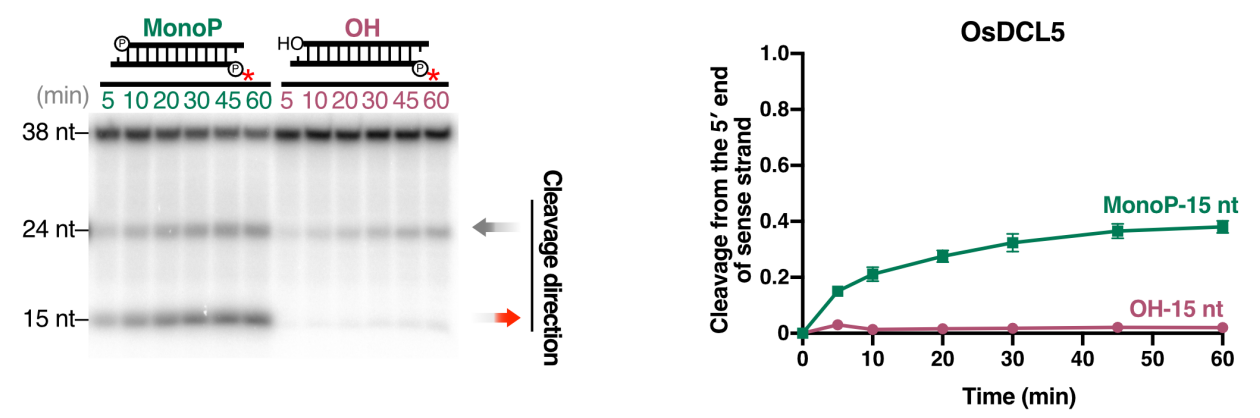
A

Chen et al., Figure 4

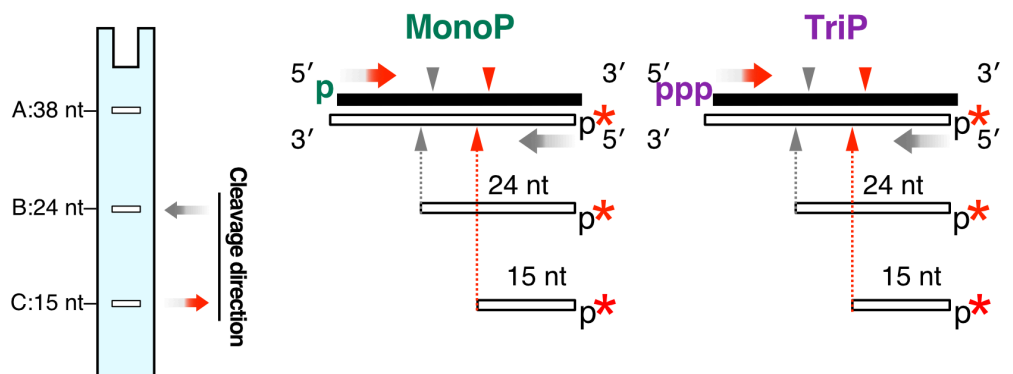

B

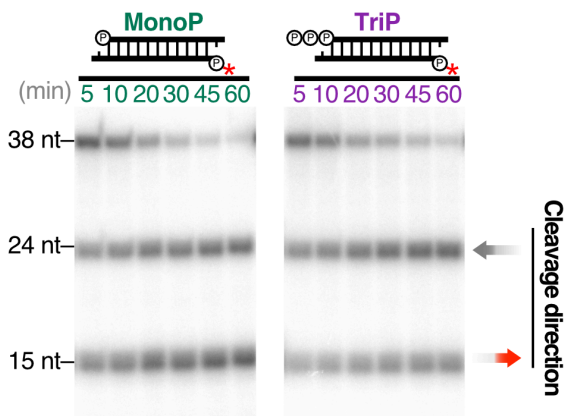

AtDCL3

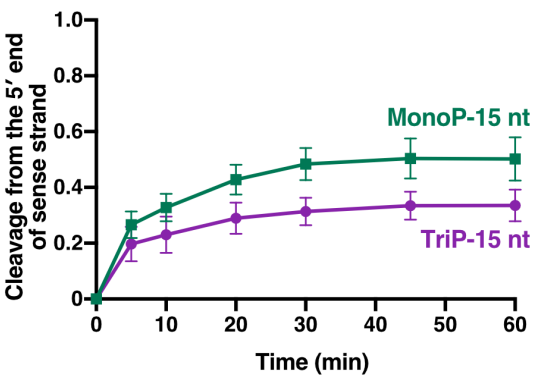

C

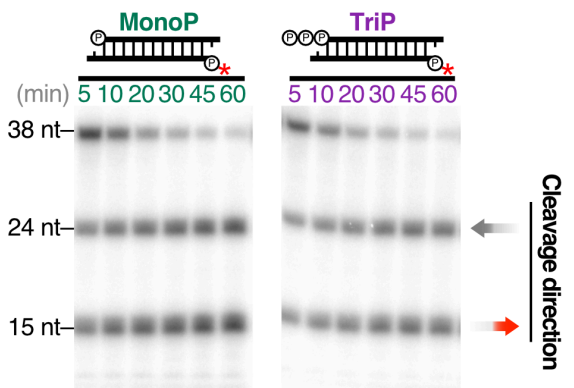

OsDCL3

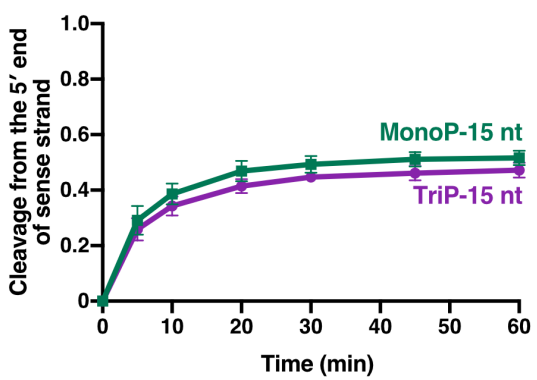

D

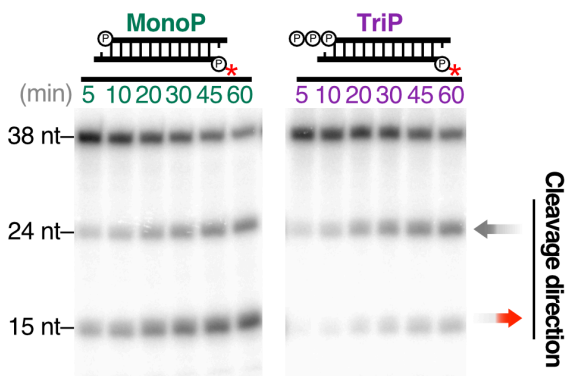

OsDCL5

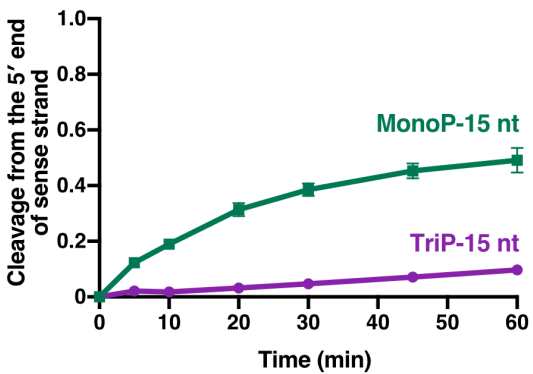




\section{Chen et al., Figure 5}

\section{A}
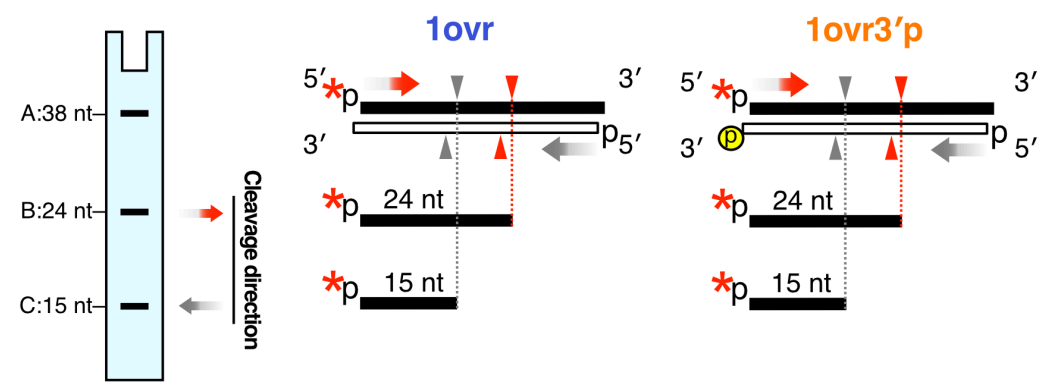

B
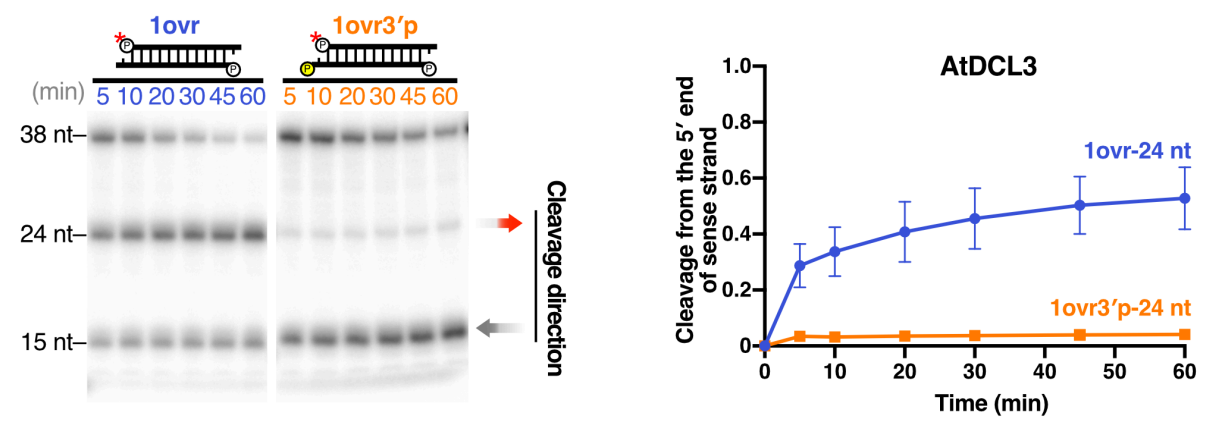

\section{C}
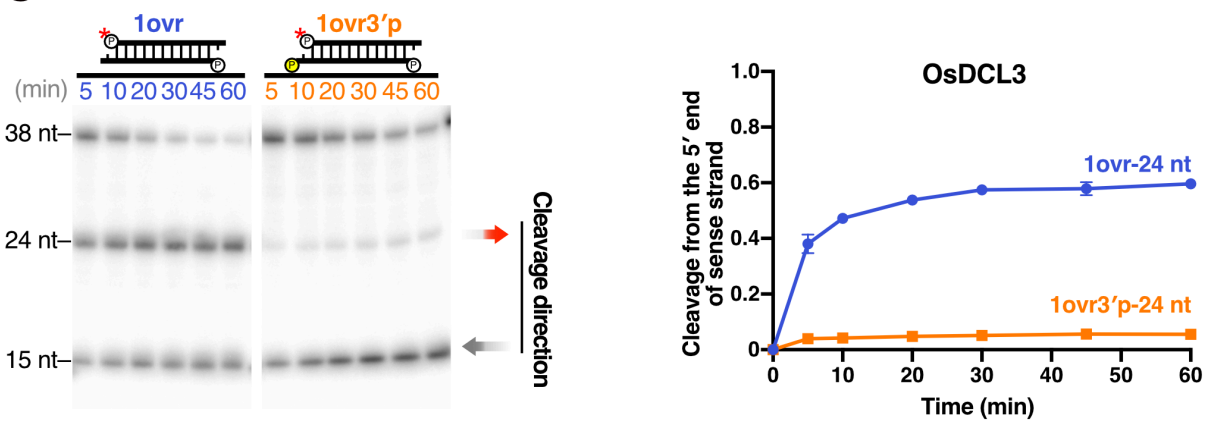

D
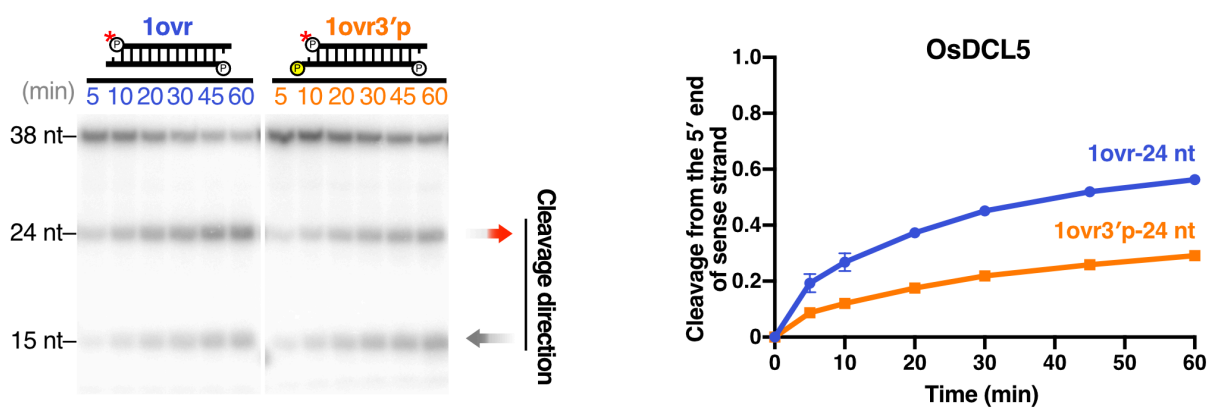
A
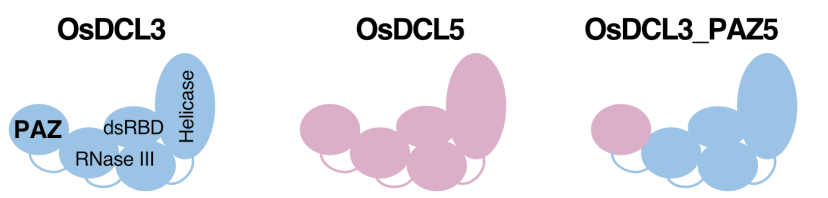

\section{Chen et al., Figure 6}

B
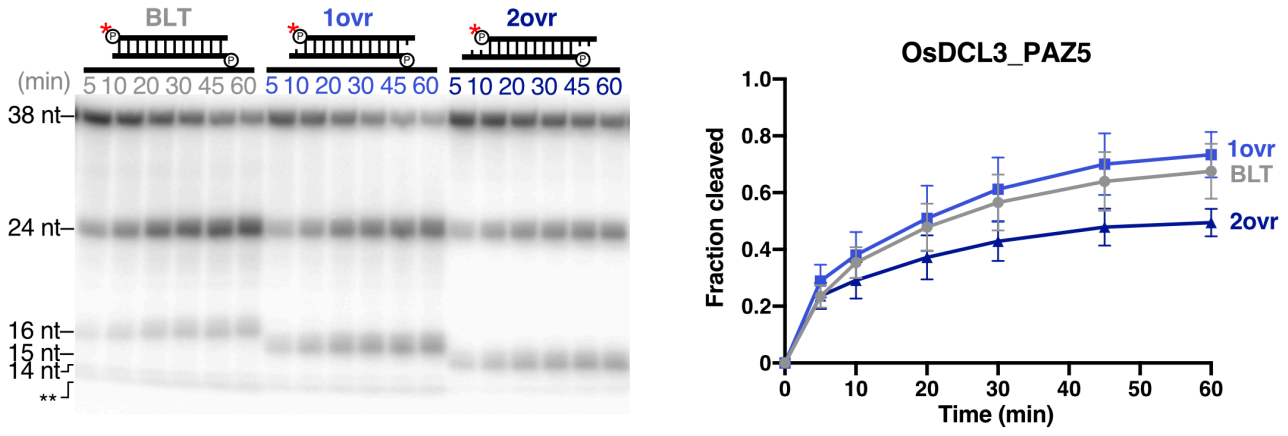

C

$16 \mathrm{nt-}$
$15 \mathrm{nt-}$
$14 \mathrm{nt}-$

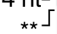

510203045605102030456051020304560

$24 \mathrm{nt}-$

(min) $\frac{\text { BLT }}{51020304560} \frac{\text { 1ovr }}{51020304560} \frac{\text { 2ovr }}{51020304560}$

$38 \mathrm{nt}-$

24 nt-

$16 n_{\substack{n+-n+-}}^{n+-}$

D

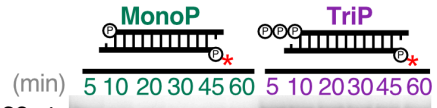

$38 \mathrm{nt}-\mathrm{sec}=\mathrm{e}$

$24 \mathrm{nt}-$

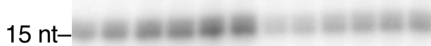

E
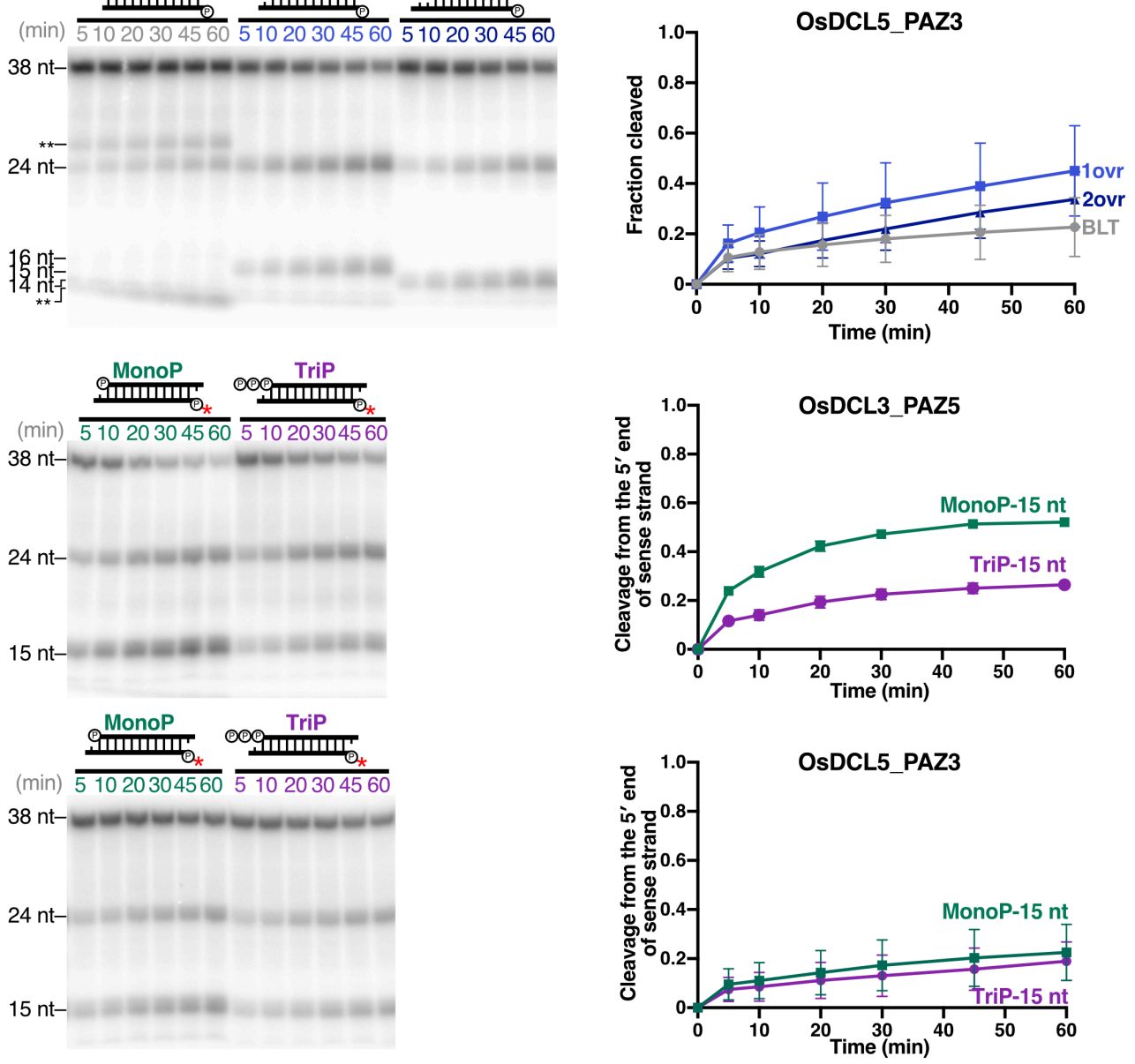


\section{Chen et al., Figure 7}

\begin{tabular}{|l|c|c|c|c|} 
& $\begin{array}{c}\text { Monocot DCL3 } \\
\text { OsDCL3 }\end{array}$ & $\begin{array}{c}\text { Eudicot DCL3 } \\
\text { AtDCL3 }\end{array}$ & Monocot DCL5 \\
OsDCL5
\end{tabular}


bioRxiv preprint doi: https://doi.org/10.1101/2021.08.02.454693; this version posted August 2, 2021. The copyright holder for this preprint (which was not certified by peer review) is the author/funder, who has granted bioRxiv a license to display the preprint in perpetuity. It is made available under aCC-BY-NC-ND 4.0 International license.

\section{Supplementary information}

720

721

Tree scale: $0.1 \longmapsto$

722

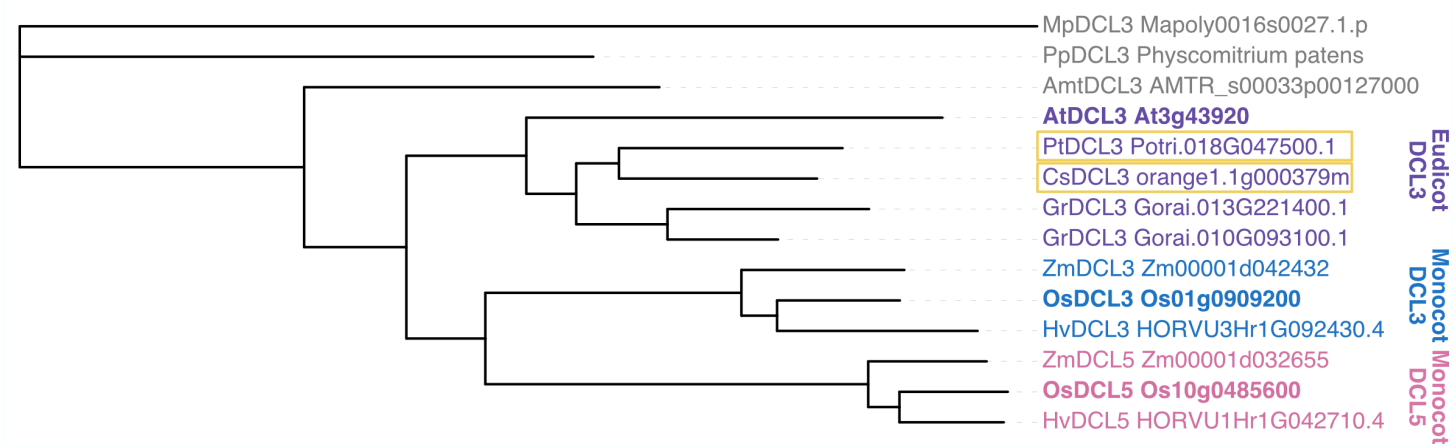

723 Supplementary Figure 1: Phylogenetic tree of DCL3 family proteins

724 The phylogenetic tree demonstrates that eudicot DCL3, monocot DCL3 and DCL5 form

725 separate clades. Eudicot plants that produce 24-nt reproductive phasiRNAs are marked

726 with yellow rectangles. Mp, Marchantia polymorpha; Pp, Physcomitrium patens; Amt,

727 Amborella trichopoda; At, Arabidopsis thaliana; Pt, Populus trichocarpa; Cs, Citrus

728 sinensis; Gr, Gossypium raimondii; Os, Oryza sativa; Zm, Zea mays; Hv, Hordeum 729 vulgare. 

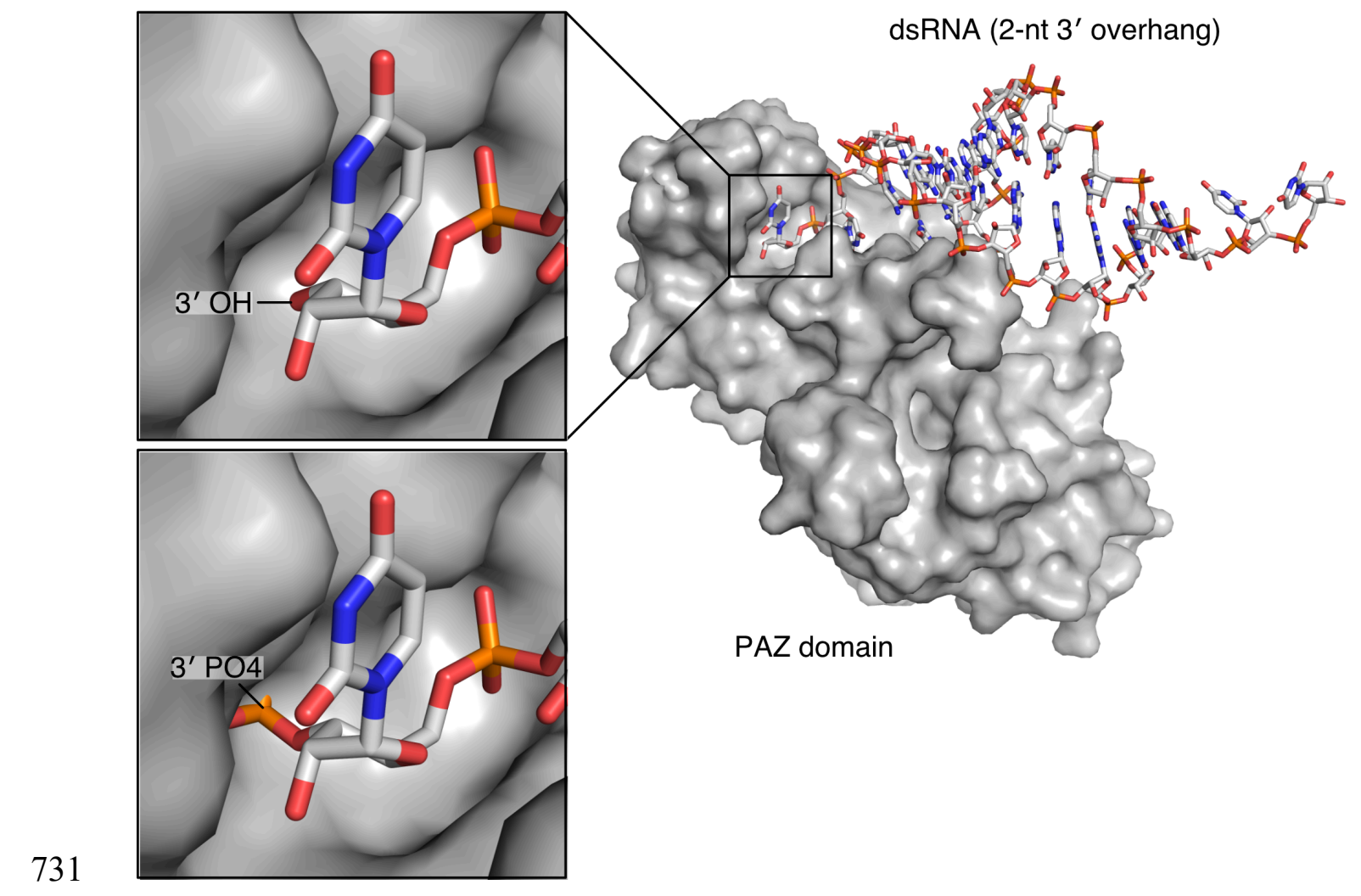

733 Supplementary Figure 2: An extra 3' phosphate blocks the binding of 1ovr substrate 734 to the PAZ domain.

735 Structure of the human Dicer fragment containing the PAZ domain bound to a 2-nt 3'

736 overhang dsRNA [Protein Data Bank (PDB) ID code: 4ngb]. The 3' hydroxyl group of

737 the dsRNA substrate is anchored to the $3^{\prime}$ binding pocket (top left). In silico replacement

738 of the $3^{\prime}$ hydroxyl group with a $3^{\prime}$ phosphate group results in a steric clash with the $3^{\prime}$

739 binding pocket (bottom left). 
A

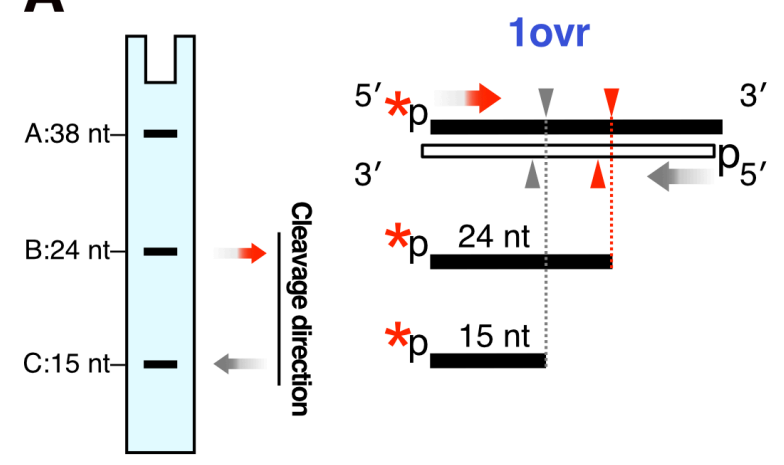

B

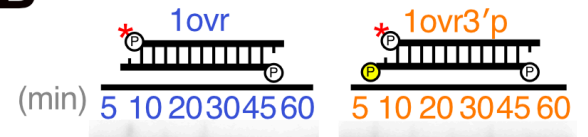

38 nt-

24 nt-

15 nt-

\section{C}

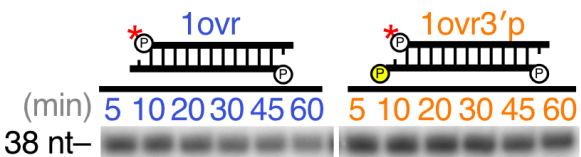

$38 \mathrm{nt}-$
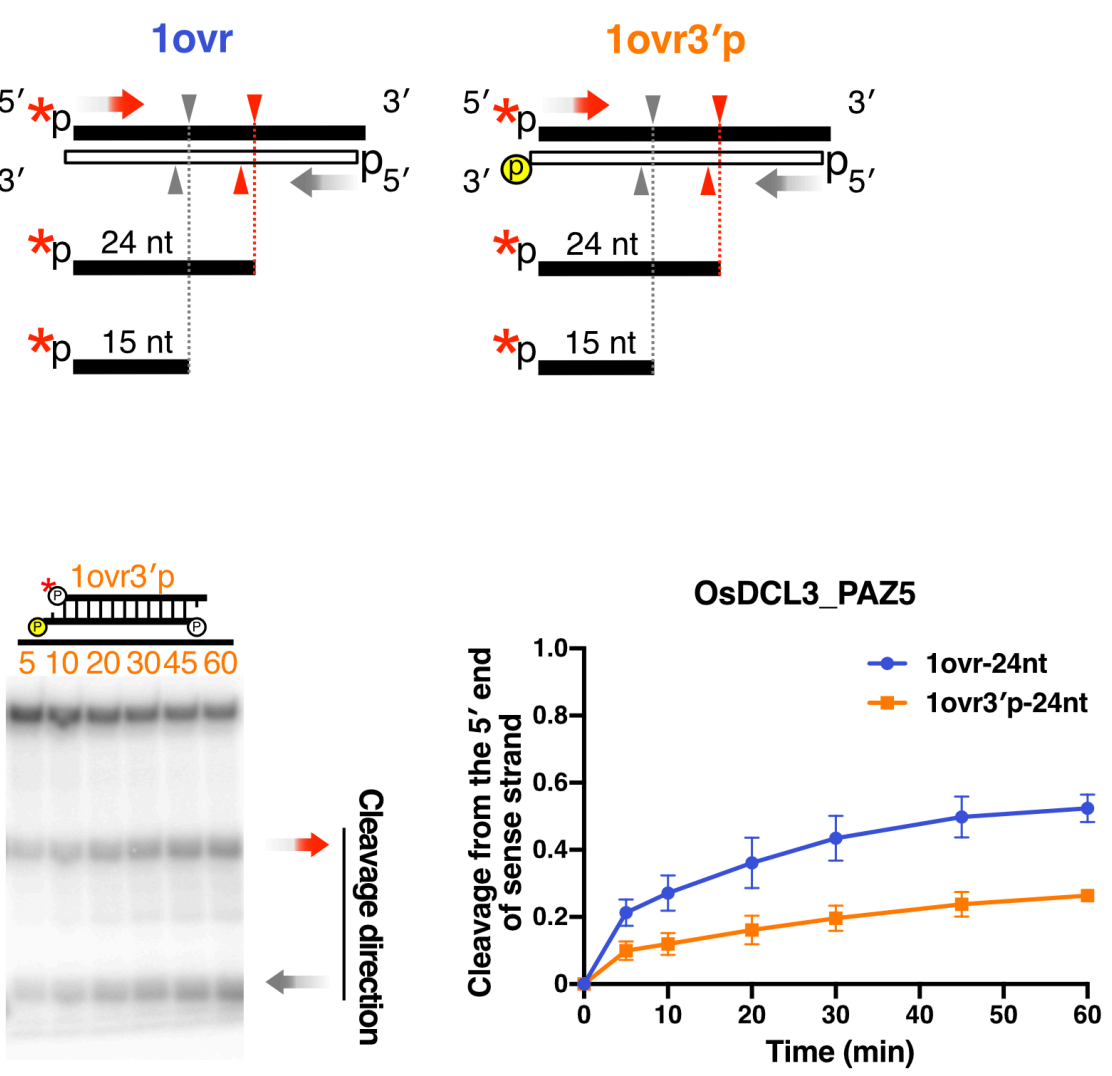

744 Supplementary Figure 3: Recognition of the 3' hydroxyl group is critical for cleavage by

\section{OsDCL5_PAZ3, but not for OsDCL3_PAZ5.}

746 (A) Dicing assays were conducted using 38-nt substrate RNAs radiolabeled on the 5'

747 monophosphate of sense strands with a lovr at both ends. An extra monophosphate group

748 was added to the $3^{\prime}$ end of the antisense strand (lovr $3^{\prime} \mathrm{p}$ ) to test the prediction that this

749 would disrupt recognition by the PAZ domain. Cleavage from the 5 ' end of sense strands 
750 (corresponding to the $3^{\prime}$ phosphate on antisense strands) results in 24-nt products.

751 (B) Left panel: A representative gel image of OsDCL3_PAZ5 dicing 1-nt overhang 752 substrates with or without a 3' monophosphate on the antisense strand (1ovr 3'p or 1ovr).

753 Right panel: Quantification of dicing reaction from the $5^{\prime}$ end of sense strands in the left 754 panel. Mean values \pm SD from three independent experiments are shown. The extra 3' 755 phosphate (1ovr 3'p) moderately reduced cleavage from the $5^{\prime}$ end of sense strand by 756 OsDCL3_PAZ5 compared with the 1ovr substrate.

757 (C) Left panel: A representative gel image of dicing assay by OsDCL5_PAZ3 cleaving 758 the 38-bp substrates 1ovr 3'p and 1ovr. Right panel: Quantification of dicing reaction 759 from the $5^{\prime}$ end of sense strands in the left panel. Mean values \pm SD from three 760 independent experiments are shown. The extra 3' phosphate (1ovr 3'p) greatly reduced 761 cleavage from the $5^{\prime}$ end of sense strand by OsDCL5_PAZ3 compared with the 1ovr 762 substrate. 
bioRxiv preprint doi: https://doi.org/10.1101/2021.08.02.454693; this version posted August 2, 2021. The copyright holder for this preprint (which was not certified by peer review) is the author/funder, who has granted bioRxiv a license to display the preprint in perpetuity. It is made available under aCC-BY-NC-ND 4.0 International license.
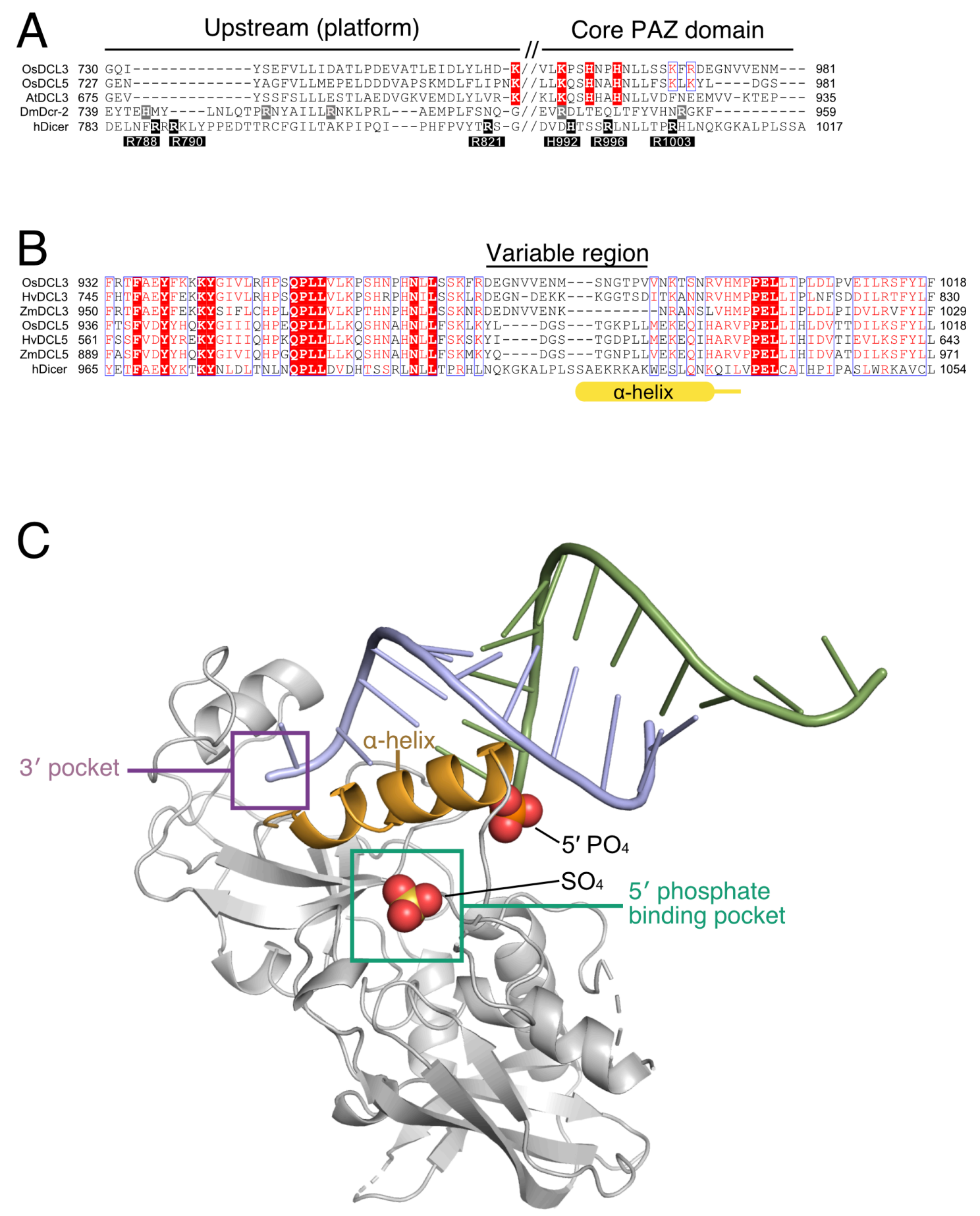

766 Supplementary Figure 4: The amino-acid sequences corresponding to the $\alpha$-helix in human 767 DICER differ significantly between monocot DCL3 and DCL5. 
768 (A) A multiple sequence alignment of the PAZ domain of Oryza sativa DCL3 (OsDCL3),

769 OsDCL5, Arabidopsis thaliana DCL3 (AtDCL3), Drosophila melanogaster Dcr-2

770 (DmDcr-2), human DICER (hDICER). White letters on black background indicate the

771 basic amino acid residues that compose the 5' phosphate binding pocket in human DICER.

772 White letters on gray and red background indicate the conserved basic amino acids that

773 correspond to the residues comprising the 5' phosphate binding pocket of human DICER

774 in Drosophila Dcr-2 and plants DCL3 family proteins, respectively.

775 Red letters indicate basic amino acids that are not completely conserved among plant

776 DCL3 family proteins. (B) A multiple sequence alignment of the PAZ domain of human

777 DICER and monocot DCL3 and DCL5. Os, Oryza sativa; Hv, Hordeum vulgare; Zm, Zea

778 mays. White letters on red background indicate amino acids that are perfectly conserved

779 across human DICER and plant DCL3 family proteins. Red letters indicate amino acids

780 that are highly conserved among human DICER and plant DCL3 family proteins. " $\alpha$ -

781 helix" highlighted in yellow shows the position of the $\alpha$-helix in the PAZ domain of

782 human DICER.

783 (C) A structure of a human DICER fragment containing the PAZ domain bound to a 2-nt

7843 ' overhang dsRNA (PDB: 4ngd). The structure includes the $\alpha$-helix that separates the 5'

785 and $3^{\prime}$ binding pockets, with the $3^{\prime}$ end of the dsRNA anchored in the $3^{\prime}$ pocket and the $5^{\prime}$

786 end released from the $5^{\prime}$ pocket. 

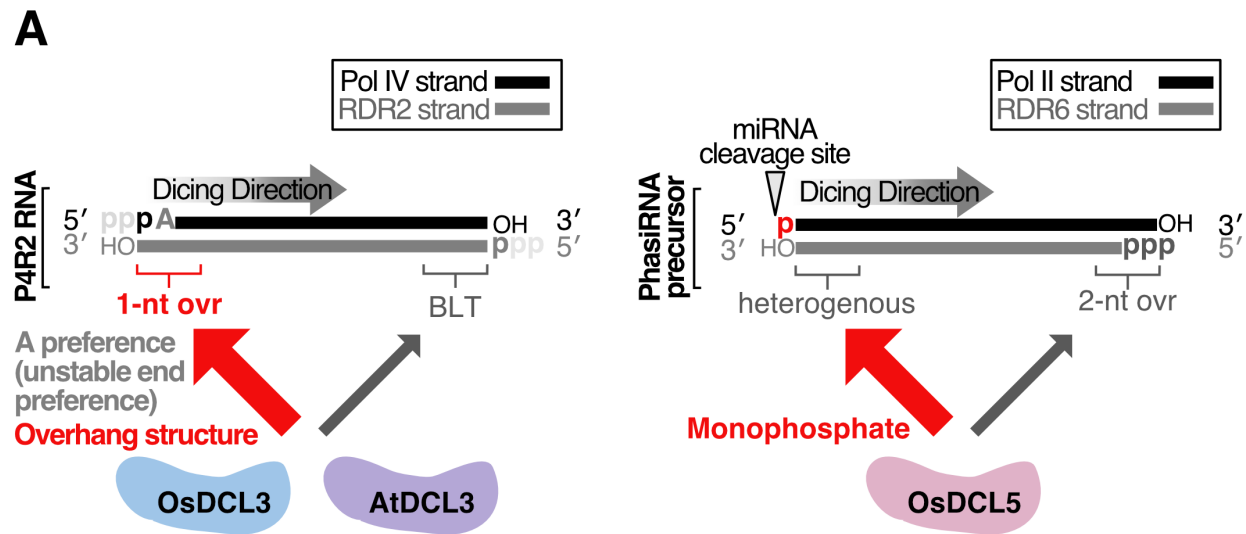

B
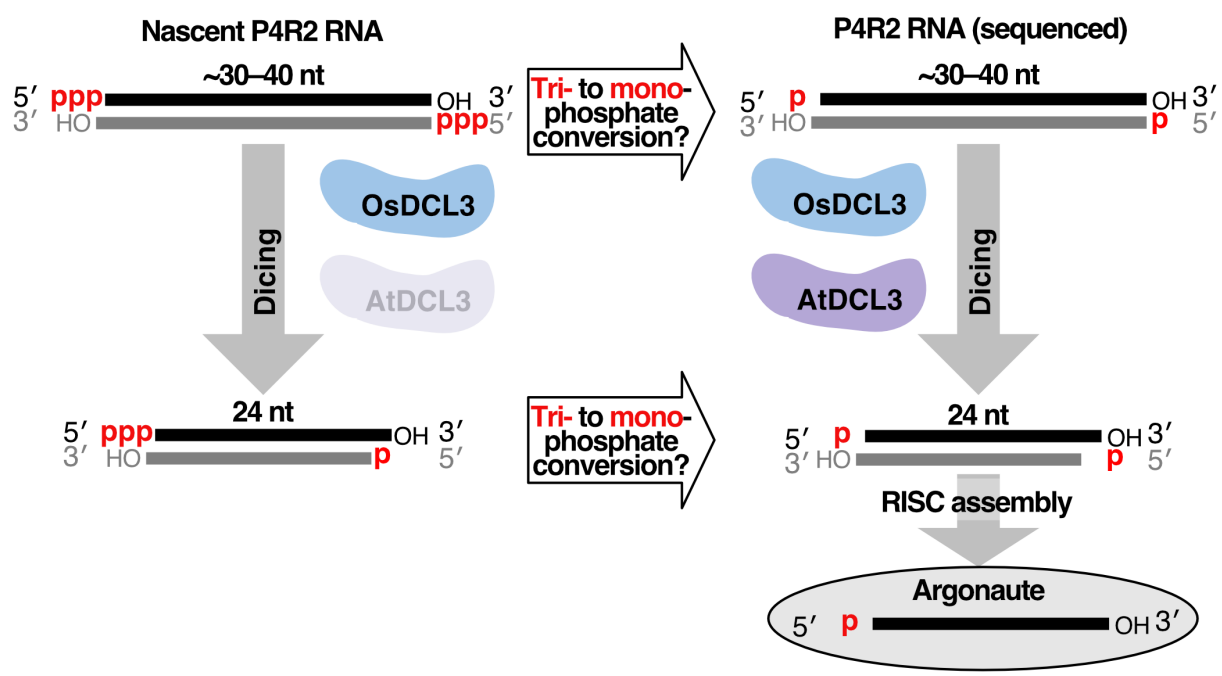

789 Supplementary Figure 5: Models for unidirectional dicing by DCL3 family members

790 and the order of tri- to monophosphate conversion and dicing by eudicot and

791 monocot DCL3.

792 (A) Models for unidirectional dicing mechanisms. AtDCL3 and OsDCL3 cleave P4R2

793 RNAs from the $5^{\prime}$ end of Pol IV strand as they prefer a 3' overhang structure and

794 unstable $5^{\prime}$ end. OsDCL5 cleaves the precursor from the $5^{\prime}$ end of the fragment due to a

795 strong preference for the 5 ' monophosphate.

796 (B) A model for the order of tri- to monophosphate conversion and dicing by eudicot

797 and monocot DCL3. Since AtDCL3 has a slight preference for 5' monophosphorylated

798 precursors, dephosphorylation prior to dicing will enhance the production of

799 heterochromatic 24-nt siRNAs in Arabidopsis thaliana. In contrast, given that OsDCL3

800 cleaves both 5' mono- and triphosphorylated dsRNAs with the same efficiency, 
bioRxiv preprint doi: https://doi.org/10.1101/2021.08.02.454693; this version posted August 2, 2021. The copyright holder for this preprint (which was not certified by peer review) is the author/funder, who has granted bioRxiv a license to display the preprint in perpetuity. It is made available under aCC-BY-NC-ND 4.0 International license.

801 OsDCL3 can effectively process P4R2 into 24-nt hc-siRNAs regardless of the tri- to 802 monophosphate conversion.

803 Table S1. The DNA oligos used in this study

\begin{tabular}{|c|c|c|}
\hline Oligo No. & Oligo name & Sequence \\
\hline 1 & AtDCL3 F & CACCATGCATTCGTCGTTGGAGCC \\
\hline 2 & AtDCL3 R & CTACTTTTGTATTATGACGATCTTGCGGCG \\
\hline 3 & AtDCL3 missing F & ТТССТСТСССТСТАAСТАTGCAGCTCAAGTATCAGAACTTGAGAGA \\
\hline 4 & AtDCL3 missing $\mathrm{R}$ & AGTTAGAGGGAGAGGAAACAATGCCTTTTCTAATGACGGCTGACGC \\
\hline 5 & OsDCL3 F & GCCGCCCCCTTCACCATGAACCCTTTAAAGAGGTCATTGG \\
\hline 6 & OsDCL3 R & CGGCGCGCCCACCCTTTCACACCTCCTGGAGTTGC \\
\hline 7 & $\begin{array}{l}\text { OsDCL5(199-927) } \\
\text { F }\end{array}$ & CACCATGGTCGCCGTCATGCTC \\
\hline 8 & $\begin{array}{l}\text { OsDCL5(199-927) } \\
\text { R }\end{array}$ & CCCATGGTATCTGGACAAGCTCTT \\
\hline 9 & $\begin{array}{l}\text { OsDCL5(912-2418) } \\
\text { F }\end{array}$ & CACCGTCCAGATACCATGGGAAAATATTGTACAG \\
\hline 10 & $\begin{array}{l}\text { OsDCL5(912-2418) } \\
\mathrm{R}\end{array}$ & CCTTTGTGCTCCACTTTTTCGA \\
\hline 11 & $\begin{array}{l}\text { OsDCL5(2389- } \\
\text { 4785) F }\end{array}$ & CACCCATGGTTCTCGAAAAAGTGGAGCA \\
\hline 12 & $\begin{array}{l}\text { OsDCL5(2389- } \\
4785) \mathrm{R}\end{array}$ & GTGCAAGGTGATTGTCGAAACAAAG \\
\hline 13 & $\begin{array}{l}\text { OsDCL5(4786- } \\
\text { 4914) F }\end{array}$ & ATACCTGACGCAACAACCATTA \\
\hline 14 & $\begin{array}{l}\text { OsDCL5(4786- } \\
\text { 4914) R }\end{array}$ & CTAAGTCTTGCATATACAGATCTTGAG \\
\hline 15 & OsDCL5 1-198_1 & $\begin{array}{l}\text { ATGGCCGACGACGAGGCTGCCGTCCTCCCGCCCCCGCCTCCGCTGCCGCCGCC } \\
\text { TT }\end{array}$ \\
\hline 16 & OsDCL5 1-198_2 & $\begin{array}{l}\text { GACCCCCTCGGGCGGAGCTGCCTGTGGGGGCGGCAAGGCGGCGGCAGCGGAG } \\
\text { GC }\end{array}$ \\
\hline
\end{tabular}




\begin{tabular}{|c|c|c|}
\hline 17 & OsDCL5 1-198_3 & $\begin{array}{l}\text { TCCGCCCGAGGGGGTCTCGACCGACTGCTGATACCACCCCTCGCACTAGCCAG } \\
\text { TT }\end{array}$ \\
\hline 18 & OsDCL5 1-198_4 & $\begin{array}{l}\text { CGATGGTGTTCCCCCGCAGCGCCGCCTCGAACACCTCCACCAACTGGCTAGTG } \\
\text { CGAGGGG }\end{array}$ \\
\hline 19 & OsDCL5 1-198_5 & AACACCATCGCGGTGCTCGACACGGGGTCCGGGAAGACCA \\
\hline 20 & OsDCL5 PAZ F & GAGCAGTTGTGGAGTTCGTATCAAG \\
\hline 21 & OsDCL5 PAZ R & ATCAGTTGTTACATCGAGATGGATAAGTAGT \\
\hline 22 & $\begin{array}{l}\text { PAZ exchange } \\
\text { OsDCL3 F }\end{array}$ & ATTTTGAGATCATTCTATTTGTTTCCGGC \\
\hline 23 & $\begin{array}{l}\text { PAZ exchange } \\
\text { OsDCL3 R }\end{array}$ & AACTGCCGTAGCTGCCG \\
\hline 24 & OsDCL3 PAZ F & AAACTTATGAGAAGGATTTATTCTGAGAATAAAAGAGAAT \\
\hline 25 & OsDCL3 PAZ R & TTCCACAGGTAAATCAAGGGGAATCA \\
\hline 26 & $\begin{array}{l}\text { PAZ exchange } \\
\text { OsDCL5 F }\end{array}$ & ATTCTGAAGTCATTTTATTTACTCCCTTCTGTAATAC \\
\hline 27 & $\begin{array}{l}\text { PAZ exchange } \\
\text { OsDCL5 R }\end{array}$ & AACTGCACCAGCACAAGATTCAAT \\
\hline
\end{tabular}

804

\section{Supplementary Methods}

806

\section{Evolutionary analysis by Maximum Likelihood method}

808 Evolutionary history was inferred using the Maximum Likelihood method and JTT 809 matrix-based model ${ }^{1}$. The tree with the highest log likelihood (-34135.57) is shown.

810 Initial tree(s) for the heuristic search were obtained automatically by applying Neighbor-

811 Join and BioNJ algorithms to a matrix of pairwise distances estimated using the JTT 812 model, and then selecting the topology with superior log likelihood value. The tree is 813 drawn to scale, with branch lengths measured in the number of substitutions per site. This 814 analysis involved 14 amino acid sequences. There were a total of 1843 positions in the 815 final dataset. Amino acid alignment by MUSCLE and evolutionary analyses were 816 conducted in MEGA $\mathrm{X}^{2,3}$. Phylogenetic tree was modified using iTOL 817 (https://itol.embl.de) $)^{4}$. 


\section{Multiple alignment of the PAZ domains of animal Dicer and plant DCL3 family}

\section{0 proteins}

821 Multiple alignment of the PAZ domains of animal Dicer and plant DCL3 family

822 proteins was performed using the PROMALS3D multiple sequence and structure

823 alignment server ${ }^{5}$.

824

825 Protein Structure Visualization

826 Protein Structure Visualization was performed by PyMol, and in silico replacement of

827 the $3^{\prime}$ hydroxyl group on the dsRNA substrate in platform-PAZ-connector cassette (PDB

828 ID: 4ngb) with $3^{\prime}$ phosphate group was done by Coot $^{6}$.

829

830

831 


\section{Supplementary References}

833 1. Jones, D. T., Taylor, W. R. \& Thornton, J. M. The rapid generation of mutation data matrices from protein sequences. Comput Appl Biosci 8, 275-282 (1992).

2. Kumar, S., Stecher, G., Li, M., Knyaz, C. \& Tamura, K. MEGA X: Molecular Evolutionary Genetics Analysis across Computing Platforms. Mol Biol Evol 35, 15471549 (2018).

838 3. Stecher, G., Tamura, K. \& Kumar, S. Molecular Evolutionary Genetics Analysis (MEGA) for macOS. Mol Biol Evol 37, 1237-1239 (2020).

4. Letunic, I. \& Bork, P. Interactive Tree Of Life (iTOL) v5: an online tool for phylogenetic tree display and annotation. Nucleic Acids Research gkab301 (2021) doi:10.1093/nar/gkab301.

5. Pei, J., Kim, B.-H. \& Grishin, N. V. PROMALS3D: a tool for multiple protein sequence and structure alignments. Nucleic Acids Res 36, 2295-2300 (2008).

6. Emsley, P., Lohkamp, B., Scott, W. G. \& Cowtan, K. Features and development of Coot. Acta Crystallogr D Biol Crystallogr 66, 486-501 (2010). 